\title{
SUR UN TYPE NOUVEAU DE CHROMOMYCOSE
}

\section{OBSERVÉ AU CANADA (TORULA BERGERI n. sp.)}

\author{
Par Louis BERGER + et M. LANGERON
}

Ce mémoire est consacré à l'étude mycologique du premier cas canadien de chromomycose. Ce cas a été découvert et décrit par le $\mathrm{D}^{r}$ Louis Berger, professeur d'anatomie pathologique à l'Université Laval, à Québec (Canada). Une première publication (Berger, Beaudry et Gaumond, 1945)' avait fait connaître l'essentiel de l'histoire du malade. L. Berger et ses collaborateurs avaient parfaitement vu qu'il s'agissait d'un champignon à phase levuriforme, différent de tous ceux qui ont été décrits jusqu'ici dans les quelque 144 ou 145 cas connus de chromoblastomycoses, ou chromomycoses; ils avaient même donné à ce champignon le nom de Candida nigra canadensis, à cause de son analogie avec le champignon décrit sous le nom de Monilia nigra par Burri et Staub (1909), C. A. Browne (1918), Maurizio et Staub (1928). Le décès inopiné et subit du Prof. Louis Berger est survenu au moment où, la documentation nécessaire étant réunie, la rédaction du mémoire était commencée.

Le Prof. Berger avait fait au $\mathrm{D}^{\mathrm{r}}$ Maurice Langeron l'honneur de lui confier l'étude mycologique de ce champignon nouveau et si intéressant. Le mémoire parait donc sous leur double signature, en hommage à la mémoire du Prof. Berger, si prématurément enlevé à sa famille, à ses élèves et à la science.

Observation(1). - Sil. A., homme de race blanche, âgé de 54 ans, savetier, n'ayant jamais quitté le Canada, entre à l'hôpital le 23 octobre 1940, pour des lésions cutanées verruqueuses et d'odeur fétide.

L'histoire de la famille ne présente pas d'intérêt médical. Le malade déclare avoir eu la fièvre typhoïde (?) à 7 ans, un abcès cervical (tuberculeux ?) à 10 ans, une pneumonie lobaire droite à 25 ans et de nouveau à 39 ans. Il a aussi souffert d'asthme jusqu'à 43 ans. Quelques mois après

(1) C'est l'observation originale, publiée en anglais par Berger, Beaudry et Gaumond, dans Canad. Med. Assoc. J., LIII, 1945, 138-143.

Ann. de Parasitologie, T. XXIV, Nos 5-6, - 1949, p. 574-598. 
son dernier accès d'asthme, débuta insidieusement une alopécie progressive, bientôt confluente, aboutissant à une dépilation totale, étendue à tout le corps. Fn même temps, il était devenu nerveux, psychiquement instable, et sa virilité avait déeliné rapidement pour aboutir finalement à l'impuissance.

En 1931, ce malade ressentit un prurit intense sur la membrane entre le $4^{\circ}$ et le $5^{\circ}$ doigts de la main gauche : ce prurit fut rapidement suivi d'une lésion maculeuse, puis verruqueuse, qui continua à se développer jusqu'à atteindre, en un an, le volume d'une noisette. En 1934, elle avait atteint le volume d'une noix et constituait une tumeur en chou-fleur, profondément suppurée, émettant un exsudat séro-purulent et fétide. Cette lésion fut enlevée opératoirement quelque part dans un hôpital, et un diagnostic histologique erroné, de carcinome à cellules épineuses (acanthosis) fut établi. La cicatrice fut traitée deux fois par les rayons X et le malade rentra chez lui.

Un mois plus tard, le prurit reparut au même endroit et fut suivi par une nouvelle poussée de croissance de la tumeur, qui envahit bientôt la totalité de l'annulaire et la plus grande partie de l'auriculaire. A peu près au même moment, une lésion semblable débuta au sommet de l'annulaire de la main droite et arriva en 2 ans au volume d'une noisette. Le malade entra en 1936 dans un autre hôpital, oủ il fut vu par le $\mathrm{D}^{\mathrm{r}}$ Gaumond et où il resta 2 mois. Une biopsie fut pratiquée pour ur diagnostic histologique et une petite portion servit à faire des cultures. A l'époque, le $\mathrm{D}^{\mathrm{r}}$ Berger pensa ètre en présence d'une «blastomycose voisine du type Gilchrist $»$, mais les cultures sur gélose de Sabouraud, pomme de terre glycérinée et carotte présentèrent, au bout de quelques jours, des colonies brun foncé, formées d'éléments levuriformes et de pseudo-mycélium, qui devinren! bientôt d'aspect carbonacé sur les deux derniers milieux.

Le diagnostic fut alors corrigé, par le $\mathrm{D}^{r}$ Louis Berger, en celui de « chromoblastomycose $»$. Un traitement iodé intensif et un autre par les rayons $\mathrm{X}$ ne donnèrent aucun résultat. La $4^{\circ}$ phalange de la main droite fut enlevée chirurgicalement et les lésions de la main gauche furent traitées par des grattages et par l'électro-coagulation.

Le malade rentra chez lui avant d'être guéri. Dans les mois qui suivirent, des lésions semblables apparurent sur le dos du pied gauche, l'avantbras gauche, la fesse droite, l'oreille droite et la joue droite.

L'odeur croissante des lésions, qui autrement étaient indolores, amena le malade à entrer de nouveau dans un troisième hôpital.

Il y fut examiné par le $\mathrm{D}^{\mathrm{r}}$ Beaudry. Physiquement, il paraissait plus àgé que son âge réel (54 ans), ressemblant plutôt à une vieille femme, mais entièrement chauve. La peau ètait blanche, lisse et brillante, les testicules petits et durs, mais autrement, à part les lésions, ce malade paraissait en bon état de santé : 4.900 .000 hématies, 8.350 leucocytes dont 48 p. 100 neutrophiles, 10 p. 100 éosinophiles, 2 p. 100 basophiles, 10 p. 100 monocytes, 30 p. 100 lymphocytes. Tous les autres examens de laboratoire de sang et d'urine ont donné des résultats normaux. 
Les lésions cutanées étaient les suivantes : $3^{\circ}, 4^{\circ}$ et $\tilde{j}^{\circ}$ doigts, ainsi que la plus grande partie du dos de la main gauche, couverts d'excroissances de couleur rose, brune ou violacée, avec des taches plus foncées, formant une masse volumineuse, verruqueuse, à aspect de chou-fleur, avec quelques éléments hémisphériques plus grands et de profondes crevasses. A quelque distance, sur l'avant-bras gauche, il y avait trois lésions semblables, du volume d'une noisette. Le sommet de l'annulaire droit, privé de sa phalangette (enlevée en 1936) était en forme de baguette de tambour et couvert par une masse verruqueuse brun-noir, mais plus foncée. L'oreille droite était en grande partie remplacée par une lésion semblable brun-noir. J.e dos du pied gauche présentait, près du premier espace interdigital, une tumeur un peu moins saillante, plus sèche et plus pâle. Sur la fesse droite était un autre nodule semblable du volume d'une noisette. Deux nodules plus petits, du volume d'un haricot et d'une cerise, sur la joue droite, ont été les derniers à apparaitre.

Il y avait, par conséquent, six localisations différentes (pl. XXVII et pl. XXVIII), celle de la main gauche étant la lésion primaire. Toutes ces tumeurs étaient plus ou moins humides, répandaient une odeur nauséabonde et variaient seulement de volume et un peu de couleur, mais étaient évidemment de même nature.

Pendant la durée du traitement, d'autres biopsies et cultures ont été faites avec diverses tumeurs, mais toutes ont confirmé le diagnostic de chromoblastomycose.

Thérapeutique. - Un traitement iodé intensif et un traitement par les rayons $\mathrm{X}$ furent essayés de nouveau pendant les premières semaines de séjour à l'hôpital, mais restèrent aussi inefficaces que ceux qui avaient été appliqués 5 ans auparavant et ne purent même pas arrêter le développement des lésions. A cause de l'aspect sénile du malade et de son insuflisance génitale, on pensa qu'un déséquilibre endocrinien pouvait avoir contribué à la pathogénicité du champignon. Aussi, on administra de fortes doses d'androstérone pendant plusieurs semaines, mais sans résultat. Un auto-vaccin fut préparé avec le champignon suivant la méthode de Méthé (Berger), mais cette vaccination resta inopérante. L'arsénothérapic et les injections de bleu de méthylène restèrent également sans succès, bien que ces dernières semblèrent d'abord arrêter l'extension des lésions. Après plus de 2 ans d'essais, les lésions furent €nlevées par électrocoagulation, grattage et intervention chirurgicale. La main gauche et l'annulaire droit furent amputés; les lésions de la joue furent enlevées avec succès par électrocoagulation; les lésions de l'oreille, de l'avant-bras et du pied furent enlevées à la fois par grattages et coagulations, mais récidivèrent plusieurs fois et n'étaient pas guéries à l'époque où l'observation a été prise. Une seule autre lésion est apparue sur la joue droite dans les quatre dernières années, pendant que le malade ètait en traitement 


\section{Examen histologique}

Les caractères sont un peu différents entre les premières biopsies et les dernières. Dans les deux cas, l'épiderme présente une hyperplasie inflammatoire avec acanthosis et papilles pénétrant profondément dans le derme. La couche cornée est macérée et parsemée de polynucléaires. Dans la première biopsie (1936), la couche supérieure du derme montre une infiltration diffuse de polynucléaires dont beaucoup sont éosinophiles, de lymphocytes et de plasmocytes, histiocytes et macrophages, tandis que dans les biopsies suivantes (1940 à 1944) l'infiltration était plus nodulaire, avec quelques microabcès, des foyers d'histiocytes avec quelques cellules géantes; dans ces biopsies, les plasmocytes prédominaient.

Le champignon se présentait sous l'aspect d'éléments levuriformes, mais cet aspect différait dans les premières et les dernières biopsies. Dans les premières ils étaient très nombreux, plus nombreux même que les cellules inflammatoires avec lesquelles ils étaient mêlés; ils étaient plutôt petits et délicats, avec une seule enveloppe mince : quelques-uns bourgeonnaient distinctement et formaient de courtes chaînes en chapelet; ils étaient surtout accumulés dans la couche supérieure, interpapillaire, du derme. Dans les dernières biopsies, les éléments parasitaires se trouvaient dans les couches moyennes ou profondes du derme ; ils étaient woins nombreux, plus volumineux, à paroi épaisse, jrrégulière, non bourgeonnants, généralement groupés et souvent à l'intérieur de cellules géantes (pl. XXXVII) ; beaucoup étaient visiblement en dégénérescence.

\section{Mycologie}

Ce champignon se développe aussi bien à la température ordinaire qu'à l'étuve. Les colonies sur gélose à la farine de maïs ou à l'infusion de foin, sur sérum glycosé de Lœffler ou sur gélose au sang restent petites (3-4 mm.) et meurent bientòt sur les deux derniers milieux. Elles sont plus grandes (4-7 mm.) sur pomme de terre glycérinée (pl. XXX, fig. 2), carotte et milieu de Sabouraud, plus grandes encore (jusqu’à $15 \mathrm{~mm}$.) sur milieux de Lœwenstein et de Petragnani. Sur Sabouraud elles sont brun foncé depuis le début et hémisphériques ; sur les autres milieux elles sont noir charbon; elles sont toujours humides, crémeuses et brillantes. A pH-7 (pl. XXXVI), elles sont surtout formées d'eléments levuriformes, avec très peu de pseudomycelium; à des $p H$ plus bas, les filaments pseudomycéliens ramifiès s'étendent à la surface et s'immergent dans le milieu ; ils portent des bouquets de blastospores. Il n'y a pas de filaments aériens (1). En eau de pomme de terre (Langeron et Talice), on obtient un dépôt de petites colonies duveteuses, le liquide tient en suspension un grand nombre de cellules de levures, isolées ou bourgeonnantes; il n'y a

(1) Non souligné dans le texte original. 
pas de voile. Ce champignon ne se développe pas en piqûre profonde en gélose ; il ne liquéfie pas la gélatine et n'a aucune action sur le lait. Il acidifie la gélose tournesolée, renfermant les sucres suivants: glycose, fructose, mannose, galactose, cellobiose, xylose, mais il n'y a jamais de fermentation avec production de gaz (1) dans les milieux liquides correspondants.

Les cultures obtenues d'une biopsie de 1944 ont donné des colonies brun foncé ou noires, se développant plus lentement, à surface sèche et rugueuse et présentant des caractères microscopiques différant de ceux des premières cultures. Le caractère fondamental est toujours l'état levuriforme, mais les cellules sont plus grandes et à paroi plus épaisse, souvent à double paroi. Leurs contours sont souvent irréguliers et beaucoup d'éléments présentent un cloisonnement interne suivant différents plans. Les structures multicellulaires qui en résultent deviennent très compliquées et sont formées de tractus plus ou moins épais, étroitement entrelacés, irrégulièrement incurvés et anastomosés, constitués par des éléments en bouquets qui se développent dans toutes les directions. Les cellules individuelles peuvent renfermer une ou plusieurs inclusions lipidiques arrondies et rappellent de très près les cellules durables (Dauerzellen) de Will ; on peut les considérer aussi comme des « cellules sclérotiales » et elles sont évidemment un variant du champignon d'abord levuriforme avec pseudomycélium (2).

En goutte pendante et en cultures sur lames, on voyait beaucoup d'éléments bourgeonnants, restant attachés à la cellule-mère et formant, par bourgeonnements successifs, des cordons moniliformes de longueur variable; ces derniers deviennent du pseudomycélium, par élongation des éléments individuels (pi. XXIX, fig. 3, pl. XXXII, fig. 2, 3 et 4 , et p]. XXXIV et XXXV). Des blastospores apicales apparaissent et donnent a leur tour naissance à des filaments pseudo-mycéliens, mais les rameaux sont peu nombreux et l'arborisation qui en résulte est, par suite, assez simple. Quelques blastospores présentent un bourgeonnement intensif de petites blastospores conidiformes, formant des amas plus ou moins volumineux de cellules facilement détachables. Il n'y a aucune trace de conidiophores ou d'aucun autre appareil sporifère spécialisé, bien que beaucoup de colonies aient été conservées vivantes pendant plus de deux ans.

En résumé, ce premier cas canadien de chromomycose est le $11^{\circ}$ pour l'Amérique du Nord. Il est caractérisé par des lésions papillomateuses durant depuis plus de 14 ans, sur la main gauche, d'où elles se sont étendues, probablement par auto-inoculation, à l'autre main, à un avant-bras, au pied, à l'oreille, à la fesse et aux deux joues. Les diverses tumeurs sont papillomateuses et semblables à celles qui ont été décrites dans les autres cas. L'iode, le bleu de méthylène, les rayons $\mathrm{X}$, les vaccins n'ont donné

(1) Non souligné dans le texte original.

(2) Nous respectons ici le texte du mémoire princeps de Perger, Beaudry et Gaumond, mais nous montrerons plus loin qu'il faut donner une autre interprétation aux éléments nommés « sclerotical cells » par les mycologues nordaméricains. 
aucun résultat; il y a eu quelques réussites avec les grattages et l'électrocoagulation, mais elles ont été suivies de nombreuses rechutes ; un doigt de la main gauche a été amputé. Au moment où cette observation a été rédigée, le malade était loin d'être guéri.

Histologiquement, les lésions ressemblaient à celles du type classique, mais présentaient une infiltration moins nodulaire dans les premières biopsies, et les éléments levuriformes étaient plus petits, plus délicats et distinctement bourgeonnants.

Les isolements, obtenus à plusieurs reprises pendant 7 ans, ont toujours donné des colonies brun foncé ou noir de charbon. Ces colonies végétaient bien et étaient crémeuses et brillantes dans toutes les biopsies, excepté la dernière en 1943, qui a fourni des colonies sèches, rugueuses et croissant lentement. Les caractères étaient différents de ceux des genres Fonsecaea ou Phialophora auxquels appartenaient tous les cas décrits antérieurement.

La prédominance de l'état levuriforme de la souche, la grande simplicité du pseudomycélium et l'absence de tout appareil sporifère spécialisé sont des caractères qui rappellent ceux des Candida. Mais dans ce genre il n'y a pas d'espèce noire, et à cause de la confusion actuelle dans la classification et la taxonomie des champignons levuriformes, nous renonçons à donner une identification définie et nous nous contentons de considérer provisoirement cette souche comme une espèce nouvelle, non nommée, d'une levure noire à aspect de Candida.

Les choses en étaient là lorsqu'un important matériel comprenant des cultures aux deux états, ainsi qu'un bloc en paraffine d'une des biopsies, fut expédié par le Prof. Berger au $\mathrm{D}^{\mathrm{r}}$ Maurice Langeron pour une étude complémentaire et, si possible, pour une détermination exacte de ce champignon.

L'état actuel des cultures est celui de colonies rugueuses du type R, formées exclusivement de Fumago.

Les premiers observateurs (Berger, Beaudry et Gaumond) avaient très bien remarqué que l'aspect des colonies de ce champignon sur les divers milieux et les caractères physiologiques de cette souche étaient très différents de ceux des souches provenant des cas déjà connus de chromomycoses et classées dans les genres Phialophora, Trichothecium, Hormodendron, Fonsecaea, etc. Se basant sur l'abondance des éléments levuriformes bourgeonnants, donnant d'abord des cordons moniliformes, puis un pseudomycelium peu ramifié, ainsi que des glomérules de petites blastospores, sans appareil sporifère défini, ils avaient été frappés par la ressemblance de ces caractères avec ceux du genre Candida Berkhout 1924, tels qu'ils ont été précisés par Langeron et Guerra 1938, sauf en ce qui concerne la couleıır des colonies. Ils avaient donc considéré à bon 
droit leur champignon comme une souche noire de Candida et lui avaient donné le nom de Candida nigra canadensis. Ils ont eu soin de rappeler aussi que Burri et Staub, C. A. Browne, Maurizio et Staub avaient déjà décrit, sous le nom de Monilia nigra, des souches saprophytes présentant à peu de chose près les mêmes caractères.

La question des levures noires se trouve ainsi posée une fois de plus : l'existence d'une souche pathogène lui donne un renouveau d'intérêt et elle prend place dans la mycologie médicale avec un représentant redoutable qui est, en pathologie humaine, un nouveau type parasitaire.

En effet, dans les cas de chromomycose décrits jusqu'ici, il n'avait pas été signalé de phase levuriforme. En revanche, il y avait toujours des appareils sporifères bien différenciés et de structure souvent complexe. C'est ce qui a été décrit sous les noms génériques de Phialophora (qui a la priorité (1), ayant été créé par Thaxter en 1915), Hormodendron et Trichosporium. Ces noms correspondent à des types morphologiques parfaitement définis.

Phialophora est caractérisé par ses phialides ventrues, pourvues d'une collerette en cupule. Ces phialides peuvent ètre isolées ou en bouquets et donnent naissance à des phialospores myxospores, c'est-à-dire non en chaînettes, comme chez les Penicillium ou Aspergillus, mais en glomérules muqueux sortant de la cupule et lui restant plus ou moins longtemps attachés. C'est, jusqu'ici, l'appareil type des chromomycoses. On le trouve, dans la nature, chez des saprophytes à thalle fuligincux, vivant sur les bois abattus ou morts, passant même dans les pulpes de bois destinées à la fabrication du papier et leur donnant une coloration bleue. Lagerberg et

(1) Au sujet de Phialophora verrucosa, il règne une certaine incertitude quant à l'auteur réel du genre et de l'espèce. Le mémoire dans lequel ce champignon a été décrit pour la première fois a été publié sous la signature de E. M. Medlar dans Mycologia (VII, 1915, 200-203). Mais en mème temps une publication analogue a été faite, d'une part par Medlar $(J$.med. res., XXXII, 1915, 507-523) et d'autre part par C.-G. Lane (1. ent. dis., XXXIII, 1915, 840-846) : dans ces deux publications, Phialophora verrucosa est présenté comme un champignon nouveau ayant causé ces accidents cutanés. Mais les deux auteurs, médecins, se réfèrent à l'autorité de Thaxter, botaniste et mycologue, qui a déterminé le champignon. Le doute ne porte pas sur le fait de la découverte, qui est d'ordre médical, mais sur le fait de la détermination et de la création des deux noms, générique et spécifique, fait d'ordre botanique et mycologique. Or c'est Thaxter qui a reconnu l'individualité du genre Phialcphora, le texte de Medlar et celui de Lane sont très explicites à cet égard. On doit donc écrire Phialophora Thaxter 1915 (in Medlar), puisqu'il n'y a pas eu de publication séparée de Thaxter. D'après Lane, c'est aussi Thaxter qui a suggéré l'épithète spécifique verrucosa. On devrait donc écrire : Phialophora verrucosa Thaxter (in Medlar et in Lane), 1915. C. Thom, dans son Naming molds, attribue le genre et l'espèce à Medlar. Conant (1937, in Mycologia, XXIX, 597-598), qui a établi l'identité de Phialophora et de Cadophora Lagerberg et Melin 1927, écrit Phialophora verrucosa Thaxter in Medlar. 
Melin avaient créé pour ces champignons le nom de Cadophora Lagerberg et Melin 1927, et Nannfeldt avait décrit en 1934 le Cadophora americana qui tombe en synonymie devant Phialophora verrucosa Thaxter 1915, comme l'a montré N. Conant (1937).

Hormodendron représente un type de sporulation qui n'est pas très répandu et qui a donné lieu à beaucoup de controverses. Il est caractérisé par un appareil ramifié, porté par un sporophore à extrémité obtuse, garnie de facettes d'insertion. De ces facettes partent des chaînettes ramifiées et articulées d'éléments sporifères dont la nature a été discutée. D'après la description donnée par l'un de nous (Langeron, 1922) et l'étude publiée par E. W. Mason en 1937, ces chaînettes sont basifuges et formées d'éléments réunis les uns aux autres par des facettes d'insertion formant disjoncteurs. Ces chaines, bien qu'extrêmement fragiles et caduques, ne peuvent se former que si la première spore reste attachée au sporophore et si les suivantes restent aussi attachées les unes aux autres, puisque la plus jeune, dernière formée, se trouve à l'extrémité distale de la chaîne. Les premières spores, qui sont aussi les plus anciennes, fonctionnent comme des parties du thalle, puisqu'elles servent à la fois de support et de canal nourricier pour les plus jeunes. Ce sont donc des thallospores et, comme elles se désarticulent, ce sont aussi des arthrospores.

Il reste a savoir si les formes ainsi cataloguées dans les chromomycoses sont réellement des fructifications du type Hormodendron. Celles-ci sont très faciles à reconnaître grâce aux facettes d'insertion fonctionnant comme disjoncteurs. Ces facettes sont très réfringentes, aussi bien sur l'extrémité du sporophore qu'aux deux extrémités des arthrospores. Ces extrémités présentent plusieurs facettes lorsque la chaîne se ramifie. Examinons par exemple les microphotographies publiées par Carrión et Silva dans leur récente monographie (1947): les fig. $7 a, 8 b, 9 a$ représentent très certainement des Hormodendron, tandis que les fig. $13 a$ et $13 b$, bien qu'elles soient étiquetées Hormodendron compactum, ne représentent pas des Hormodendron, même en admettant que l'accroissement des rameaux soit basifuge, car l'insertion des articles a lieu par une large base et on ne voit pas trace de disjoncteurs. Pourtant, dans la nature, les sporophores et les spores des Cladosporium et Hormodendron sont très faciles à reconnaître à l'épaisseur et à la réfringence des facettes d'insertion.

Bien que nous n'ayons pas eu à notre disposition de cultures d'Hormodendron compactum originales, nous pensons que ce champignon, dont l'individualité paraît certaine, n'appartient pas au genre Hormodendron. 
Dans la première monographie de Carrión, publiée en 1942, il y a aussi de très bonnes microphotographies. La fig. 4 représente très exactement les types Hormodendron ( $a$ et $b$ ) et Phialophora ( $c$ et $d$ ). Il en est de mème, dans la fig. 5 , de $b, e, f, g, j$ pour Hormodendron et de $k, l$ pour Phialophora, mais $5 c$ est confus et non démonstratif ; dans la fig. 6, $a$ et $b$ montrent bien Phialophora. Simson, Harington et Barnetson (1943) ont donné une remarquable représentation d'un Hormodendron très ramifié à nombreux pinceaux (fig. 5-7 et 12-13).

Ce type d'appareil conidien (1) n'est pas limité aux champignons à thalle fuligineux (dématiées) : il existe aussi chez les Mucédinées. Mlle H. A. Diddens a décrit en 1931 le genre Hyalodendron pour un champignon (H. lignicola) isolé en Suède par E. Melin, de pulpes de bois atteintes de la maladie bleue. Les chainettes de spores sont articulées et basifuges, exactement comme chez les Hormodendron, mais elles sont incolores. Mlle Diddens a joint aux Hyalodendron H. album (Dowson 1924) (= Cladosporium album Dowson 1924). La même structure se retrouve chez H. pirinum Goidanich 1935 et chez les macroconidies de Mycosphrrella punctiformis, forma tiliae, d'après E. W. Mason 1937.

Mais la validité d'Hormodendron est douteuse. C. Thom (1940), dans une revue critique intitulée : Naming molds, a montré, une fois de plus, qu'il n'y a pas de différence réelle (2) entre Hormodendron et Cladosporium et que ce dernier a la priorité. Nous sommes d'accord pour suivre cet avis et pour nommer Cladosporium tous les appareils conidiens à éléments basifuges, isolés ou en chaìnettes plus ou moins ramifiées et présentant les facettes d'insertion et de disjonction caractéristiques (3).

(1) Hormodendron algeriense Montpellier et Catanei 1927 et $H$. langeroni da Fonseca, Leão et Penido 1927, isolés tous deux de dermatites non verruqueuses, sont de vrais Hormodendron, dont les articles sporiformes en chaines basifuges portent les facettes caractéristiques.

(2) Il semble que le seul critère classique permettant de distinguer Hormodendron de Cladosporium soit, chez ce dernier, l'existence, dans la spore, d'une cloison qui n'existe pas chez le premier. Cette distinction subtile parait avoir perdu, heureusement, toute valeur. Lindau a déerit en 1907, dans la Rahenhorst's hryptogamen Flora, Dic Pilze, VIII, p. 699, $\mathrm{n}^{\circ} 1385$, un Hormodendron resina pour une dématiée trouvée sur résine de sapin dans le Sachsenwald, près de Hambourg. Ce champignon a ćté retreuvé par Christensen, Kaufert, Schmitz et Allison (Amer. J. bot., XXIX, 1942, 552-558) largement répandu aux Etats-Unis sur les bois imprégnés de créosote et de coaltar. Dans ce travail récent, on sépare encore Hormodendron de Cladosporium par la présence d'une cloison dans la spore de ce dernier.

(3) M. A. Brett, The problem of Cladosporium herbarum. Brit. mycol. Soc. Trans., XXX, 1948, 141-151. Ce problème n'est pas résolu. Pour nous il n'existe pas : il n'y a rien de commun entre Pullularia pullulans et le groupe Hormodendron-Cladosporium. Ils sont séparés par la présence ou l'absence des facettes d'insertion et Hormodendron n'est qu'un Cladosporium plus rameux comme le montre bien la figure publiée par Simson, Harington et Barnetson (1943) et citée plus haut. L’appareil conidien de Pullularia n'est jamais basifuge ! 
Trichosporium. - Ce que l'un de nous désigne sous ce nom, depuis sa publication princeps de 1929 , est un apparcil conidien très différent des deux précédents. C'est un type tout à fait net de ce qu'E. W. Mason a décrit dans la suite (1933) sous le nom de raduíaspores; on le rencontre chez un assez grand nombre de champignons, notamment chez les sporotrichés. Il est constitué typiquement par un renflement terminal en massue hérissée de denticules portant chacun une conidie.

On persiste à confondre les genres Trichosporium et Acrotheca. Carrión (1942) écrit (p. 435) : "When Ota (1928) and Langeron (1929) erroneously placed Pedrosoi among the Trichosporium, they were evidently impressed by the Acrotheca-like clusters, which were mistaken for Trichosporium... ». C'est une opinion qui, malheureusement, ne repose que sur des affirmations. Pour s'en convaincre, il suffit de jeter les yeux sur la figure donnée par Preuiss, auteur du genre Acrotheca. Il n'est pas nécessaire de posséder le mémoire original de cet auteur, car cette figure est très bien reproduite dans des ouvrages classiques, tels que la Rabenhorst's Kryptogamenflora (VIII, 1907, p. 1255, $\mathrm{n}^{\circ} 1.256$ ) et les Pflanzenfamilien d'Engler et Prantl. Si l'on veut bien se donner la peine de les consulter, on verra qu'Acrotheca développe aussi un appareil conidien du - type radula-spores, mais cet appareil surmonte un conidiophore très diffèrencié, émis par un thalle végétatif à peine visible ou évanescent. Au contraire, chez les Trichosporium, la massue hérissée de radulaspores se développe à l'extrémité de rameaux non différenciés, faisant partie d'un thalle végétatif bien développé, formant feutrage. Nannfeldt (in Melin et Nannfeidt, 1934) a donné aussi d'excellentes figures d'appareils à radula-spores et son Rhinocladiella atrovirens, des pulpes de bois bleuies en Scandinavie, rappelle étrangement Trichosporium pedrosoi (1).

Il faut donc se décider à admettre le type d'appareils conidiens à radula-spores et à abandonner le terme générique Acrotheca qui ne répond pas à la réalité des faits. Les radula-spores du type Trichosporium sont bien représentés par Carrión (1942) (fig. $4 e$ et $f$; fig. $5 a, d, i, k$; fig. $6 c, d, e, f, g)$. F. D. Weidman et L. H. Rosenthal, dans leur excellente mise au point (1942), désignent les têtes de radula-spores par le terme significatif de «pseudo-Acrotheca »!

Il se trouve que Terra, Torres, da Fonseca et de Arêa Leão (1922), auteurs de l'erreur initiale concernant Acrotheca, ont donné, à la p. 366 de leur mémoire, deux très bonnes figures, bien qu'un peu

(1) D'ailleurs Vuillemin (1911) a rejeté Acrotheca qu'il considìre comme très voisin des Ramularia et bien différent des Gomphinaria et des Acrothecium. 
petites, d'un appareil conidien à radula-spores du type Trichosporium, tel qu'il a été décrit ensuite par Mason, Nannfeldt et nousmême. Mieux encore, Aroeira Neves (de Bello Horizonte) a publié, toujours dans Brasil-medico (19 janvier 1929), et bien entendu sous le nom d'Acrotheca pedrosoi, un remarquable dessin, peut-être le meilleur qui existe, de l'appareil conidien d'un Trichosporium, isolé d'une onychomycose généralisée. Cette observation, qui parait peu connue, est très intéressante aussi au point de vue de l'origine saprophytique de ce champignon. P. Negroni, sous le nom de Fonsecaea, a publié un mélange d'appareils Trichosporium et Hormodendron.

Nous ne pouvons souscrire à l'opinion émise par C. Thom (1940) dans son Naming molds: d'après lui, la forme Acrotheca (notre Trichosporium) ne serait qu'une réduction progressive de la ramification du type Cladosporium jusqu'à une simplification ultime en bouquets denses de spores primaires, réunies sur les extrémités en massue de filaments fertiles; quelques cellules formeraient alors une courte série de bourgeons du type Cladosporium. En somme, réduction progressive du type complexe Cladosporium vers le type «Acrotheca »! Ceci provient de la méconnaissance à peu près générale de la structure à facettes des rameaux conidiens basifuges du type Cladosporium, alors 'qu' «Acrotheca » a la structure radula-spores.

Ajoutons que l'état Trichosporium des agents des chromomycoses a été décrit par l'un de nous, dès 1929, sur le matériel original, isolé par Pedroso et rapporté par E. Birumpt du Brésii, en cultures vivantes. Acrotheca, qui n'est pas défendable, ne peut même pas être considéré comme synonyme. La structure basifuge à facettes des Cladosporium-Hormodendron a été décrite et figurée par le même dès 1913 pour $H$. fontoynouti.

Ce qui complique la question et la rend très obscure pour ceux qui ne sont pas familiarisés avec le polymorphisme et la plasticité des champignons, c'est que ces trois formes conidiennes peuvent soit apparaître séparément dans des souches diverses, soit coexister chez la mème souche, donc sur le même thalle et sur un même filament de ce thalle. Dès 1937, N. F. Conant a reconnu l'identité de Cadophora americana Nannfeldt 1934 et de Phialophora verrucosa Thaxter 1915. Cadophora a été créé en 1927 par Lagerberg et Melin pour des saprophytes du bois, très répandus dans les régions forestières et qui causent le bleuissement des pulpes de bois. Cadophora doit donc tomber en syronymie devant Phialophora qui a la priorité et tous les Cadophora (8 espèces) deviennent des Phialophora. Déjà en 1933, Wilson, Hulsey et Weidman, étudiant un cas observé 
ANNALES DE PARASITOLOGIE

T. XXIV, $\mathrm{N}^{\text {os }} 5-6,1949$
Planche XXVII

(Mémoire Berger et LANGeron)

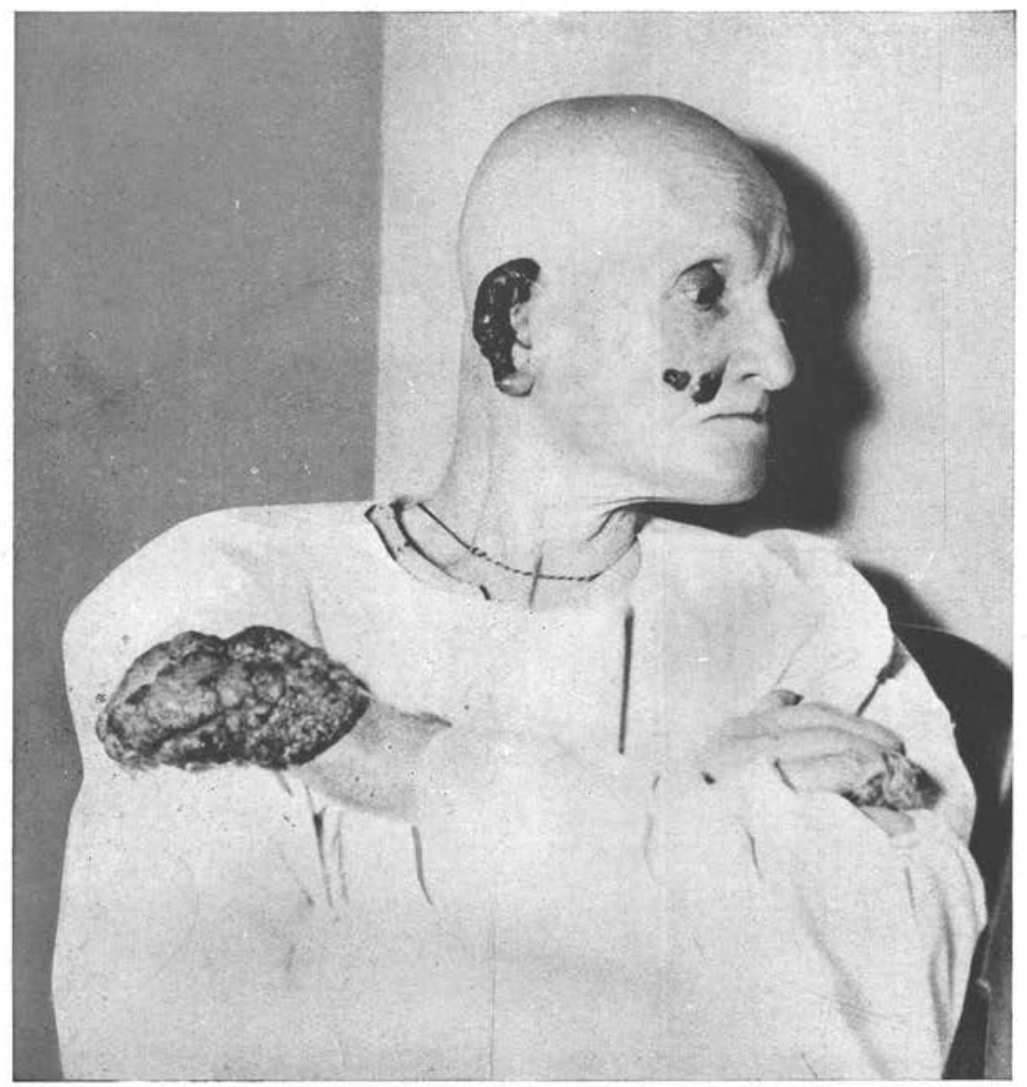

Aspect du malade en 1940, montrant 4 des 7 localisations développées en 12 ans : main gauche (début des lésions), medius de la main droite, oreille droite, joue droite. 

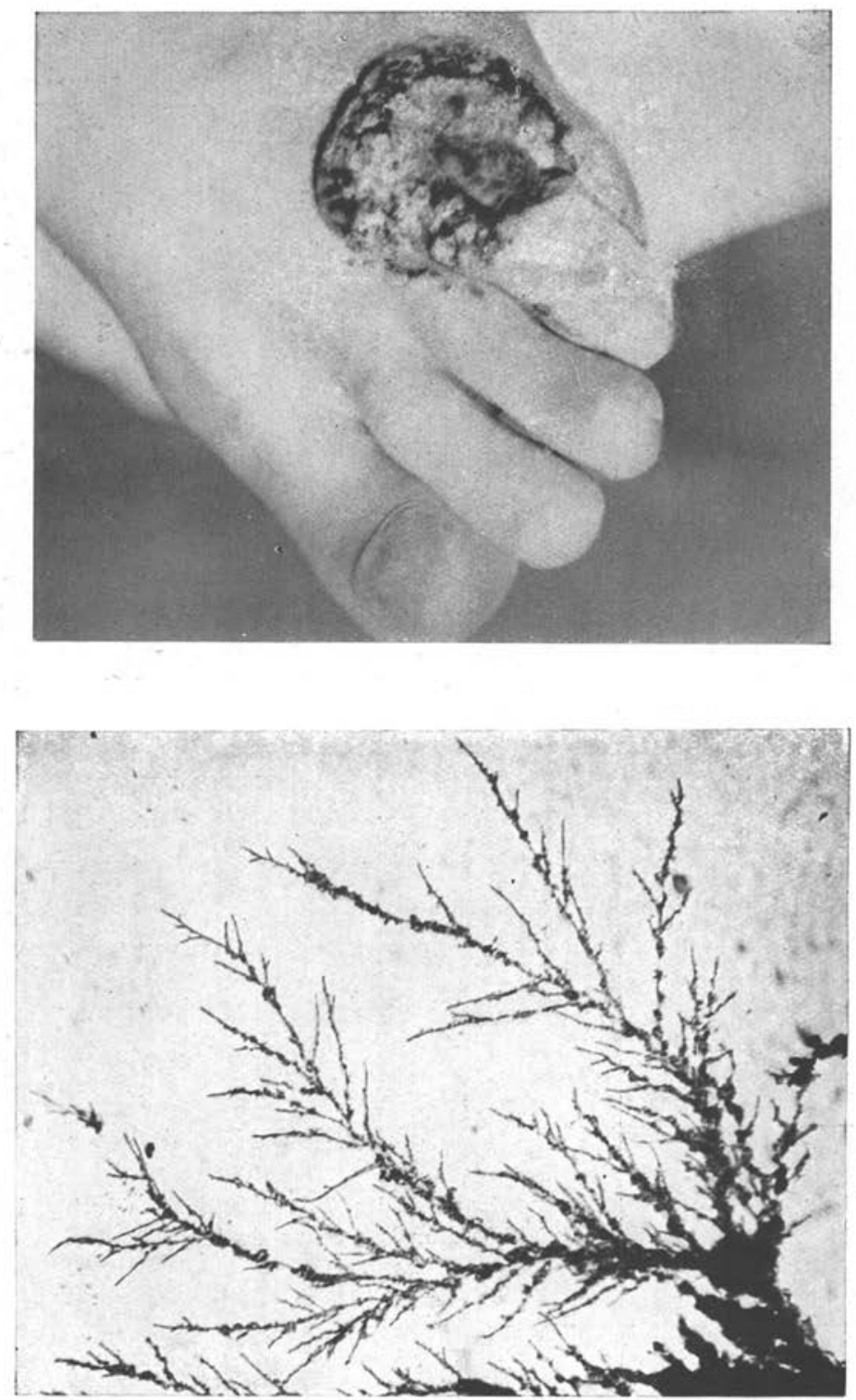

En haut, lésion du pied gauche ; en bas, culture de 40 jours de la forme levure, sur lame gélosée. $\times 86$. Microphotographie. 

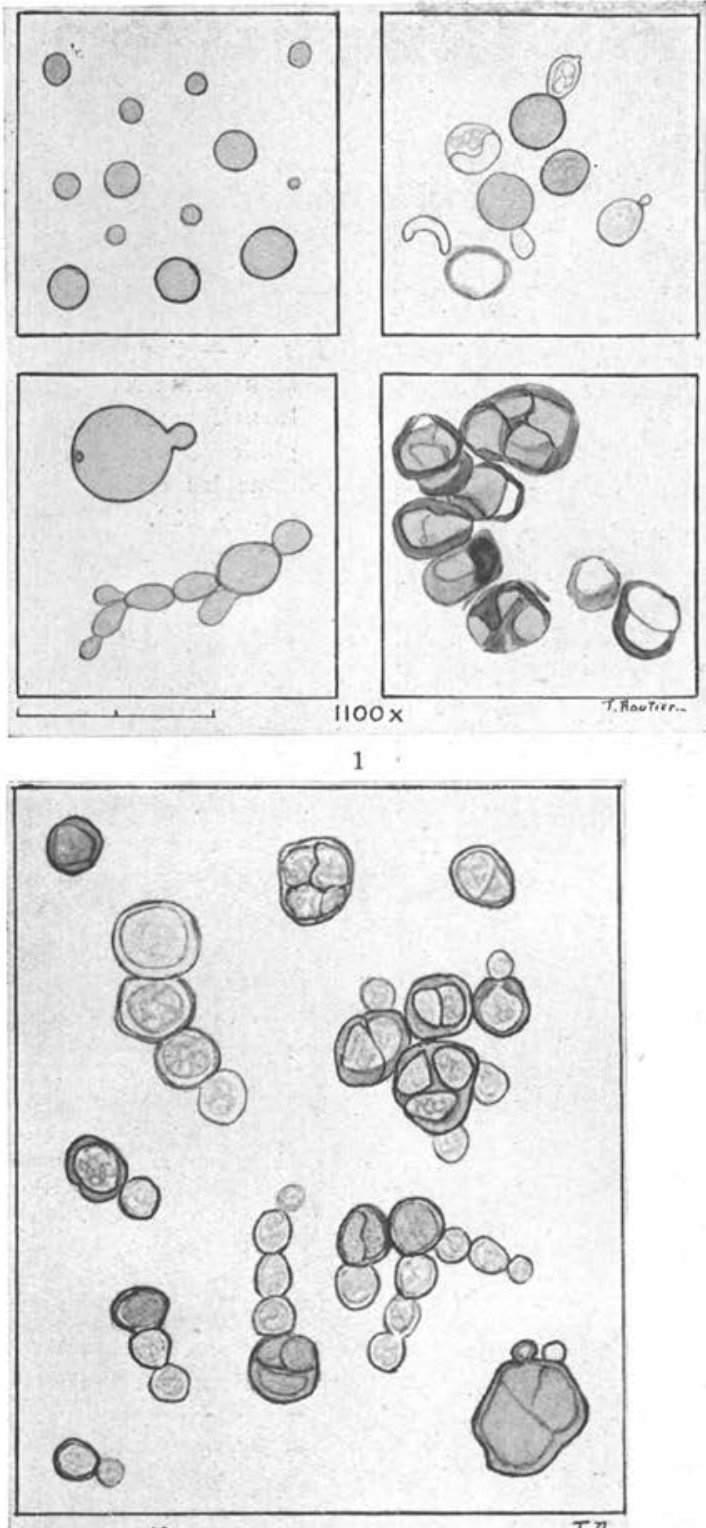

$10 \mu$

T.R.-

2

3

FiG. 1. - Formes levures (en haut et en bas à gauche) et formes Fumago (en bas à droite), dans la $1^{\text {re }}$ biopsie de la lésion.

FIG. 2. - Colonies fumagoïdes sèches (type R) de 4 mois, écrasées dans la glycérine.

FIG. 3. - Eléments levuriformes et pseudo-mycelium en eau de pomme de terre (bleu coton au lactophénol). 


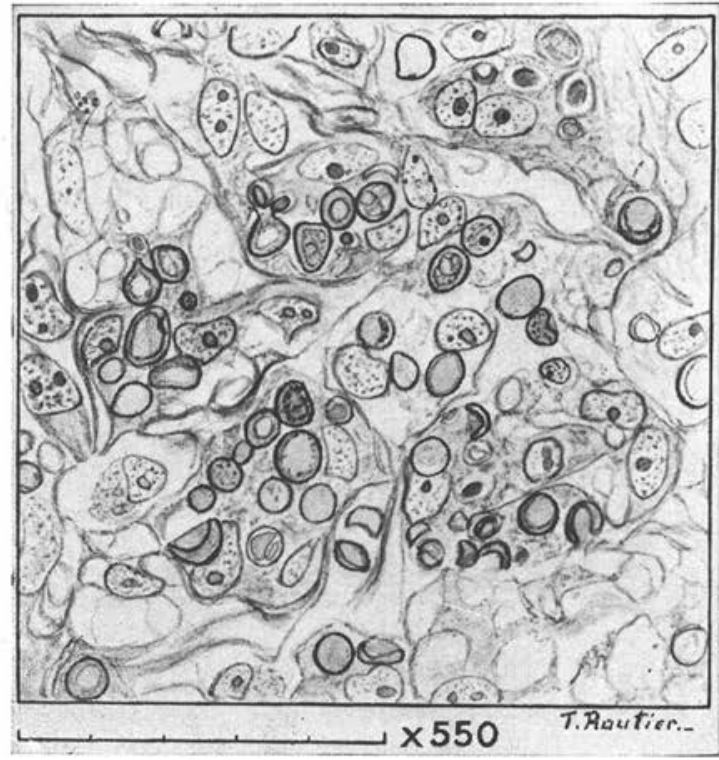

Fig. 1. - Coupe de la Iêsion primaire. Eléments levuriformes et fumagoïdes libres ou inclus dans les cellules géantes. $\times 550$.

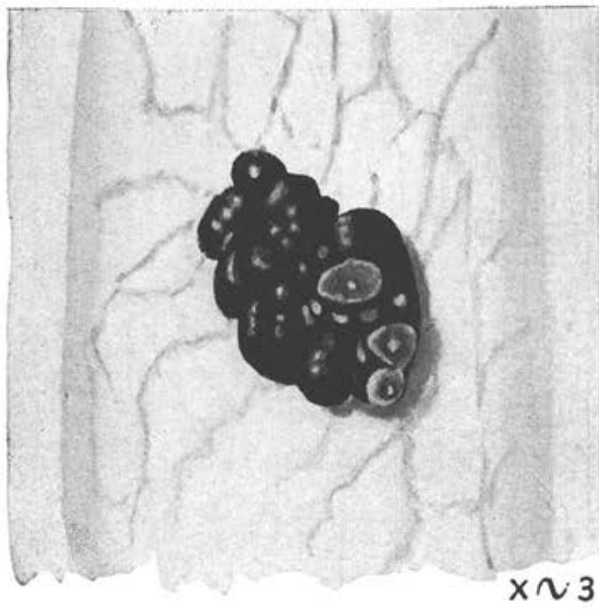

2

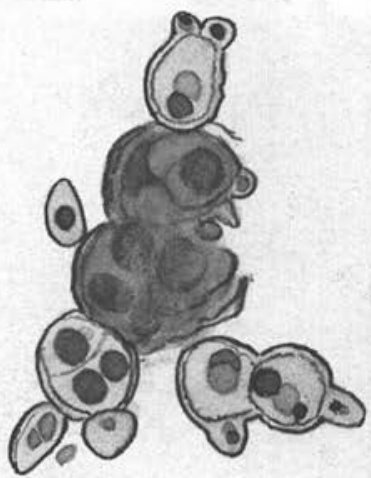

$1540 x$

Fig. 2. - Colonie lisse et brillante (type S) de 4 mois sur pomme de terre. $\times 3$. Fig. 3. - Fragment de colonie sèche (type R) de 4 mois, écrasé dans le lactophénol. Eclosion de Fumago. 
au Texas, avaient suggéré que les états Trichosporium (Acrotheca) et Phialophora pourraient être deux formes conidiennes d'une même espèce ou d'un même genre. Cette hypothèse a été confirmée et élargie par Carrión et Emmons (1935), puis par Martin, Baker et Conant (1936), enfin par Conant et Martin (1937).

Ces faits, qui concordent avec ce que l'on sait sur les macro- et microconidies, sont maintenant admis par tous les auteurs, et on les trouvera présentés dans la monographie très complète publiée par A. L. Carrión et M. Silva dans le remarquable volume de Nickerson (Biology of pathogenic fungi, 1947), avec de belles illustrations.

Fumago. - Mais un point a été négligé, ou plutôt mal interprété par tous ceux qui ont étudié les chromomycoses : c'est la réduction morphologique parasitaire. Le champignon, quelle que soit la disposition de son appareil conidien, subit cette réduction dans les tissus de l'homme, ou de l'animal, infecté. Dans les lésions des chromomycoses on trouve en effet, soit dans les cellules géantes, soit à l'intérieur de micro-abcès, soit même libres dans les tissus, des éléments pigmentés, plus ou moins sphériques, isolés ou en amas, non bourgeonnants, souvent divisés par des cloisons diamétrales (1), donc se multipliant par scission. Ces éléments ont été décrits et figurés maintes fois (2). De très bonnes microphotographies ont été publiées par Weidman et Rosenthal (1941, fig. 2, p. 65) et par Carrión (1942, fig. 3 , p. 431). On ne les trouve, dans les nombreuses publications où ils sont cités, que sous le nom, tout à fait impropre, de « cellules sclérotiales » (sclerotial cells) qui leur a été donné par les mycologues nord-américains.

En réalité, ils sont connus depuis très longtemps sous le nom de Fumago (3), ou formes fumagoïdes. Ils constituent la forme réduite des champignons des fumagines, dématiées habituellement filamenteuses, produisant à la surface des végétaux, principalement des feuilles, un dépôt noirâtre, carbonacé, ressemblant à de la suie ( $f u$ mus, fumée, suie). Ces champignons, à pigment fuligineux (fuligo,

(1) F. X. Skuplensk1, faisant agir du goudron sur Cladosporium herbarum, a obtenu des amas de cellules fumagoïdes ressemblant aux Fumago des chromomycoses (Acta Soc. bot. Poloniae, XI, 1934, 541-551, 2 pl.). Medlar (1915) les avait déjà obtenues sur gélose ascîte et sérum glycosé de Löffler et Mackinnon (1936) sur Czapek, ainsi que Moore et Mapother 1940.

(2) M. E. Laurent, loc. cit., p. 599. - L. Planchon, loc. cit., p. 37-38 et pl. IV, fig. $4,8,11,12$. Voir aussi la belle figure des frères Tulasne, reproduite dans lé Rahenhorst's Krypt. Flora, Pilze, IX, 1910, p. 267.

(3) Fumago a été décrit par Persoon (1801). Le binòme Fumago vagans Persoon a servi pendant longtemps à désigner les champignons des fumagines. Ces champignons sont maintenant réunis dans la famille des Capnodiacées, qui a pour type le genre Capnodium (Ascomycètes dothidéales).

AnN, de Parasitologie, T, XXIV, $\mathrm{N}^{\text {os }}$ 5-6, - 1949. 
suie), appartiennent aux genres Alternaria, Cladosporium, Capnodium, Teichospora, Phialophora, Pullularia, Rhinocladiella, etc. Ce sont des saprophytes vivant sur le bois et les végétaux forestiers.

Les formes fumagoïdes ne sont pas des chlamydospores morphologiques, car elles ne présentent pas les caractères des appareils d'enkystement. C'est seulement un thalle réduit. On ne le trouve pas seulement dans les lésions des chromomycoses, mais aussi en abondance dans la nature. Il suffit d'examiner attentivement des enduits de fumagine. On ne peut done, avec Carrión et Silva (1947), les considérer dans certains cas comme de véritables chlamydospores.

Connus depuis longtemps des anciens botanistes, ils ont été étudiés plus récemment par Laurent (1888), puis par Planchon (1900) qui, dans un mémoire peu connu et même méconnu, en a donné d'excellentes figures en couleurs, à propos de Pullularia pullulans, de Cladosporium herbarum et des Alternaria. La présence constante de ces éléments dans les lésions de tous les cas décrits jusqu'ici de chromomycoses est un argument très puissant pour confirmer l'origine saprophytique et forestière de ces maladies.

Le cas que nous étudions ne présente de commun avec les précédents déjà décrits que les nombreux Fumago présents dans les lésions. Pour le reste, aucune des trois formes conidiennes : Phialophora, Hormodendron, Trichosporium, n'a été observée depuis les huit années que les cultures sont entretenues et suivies.

Par contre, surtout dans les cultures premières, est apparu, en abondance et exclusivement, un état levuriforme blastosporé. Il s'agit donc, jusqu'ici, uniquement d'une «levure noire ».

\section{Les levures noires}

Il existe des levures noires, c'est-à-dire des champignons levuriformes donnant des colonies crémeuses, colorées en brun foncé, plus ou moins olivâtre, ou même en noir pur, par un pigment fuligineux. L'examen microscopique de ces colonies révèle l'existence de nombreuses cellules levuriformes et d'une filamentisation. Celle-ci est, en général, constituée par un pseudomycelium, c'est-à-dire par des filaments formés de cellules de levures, nées par bourgeonnement, plus ou moins allongées, et accolées bout à bout par leurs pôles. Ce sont de faux filaments (pseudomycelium) puisqu'il ne s'agit pas d'un tube cloisonné. Ce que beaucoup d'auteurs ont pris, chez les levures blanches, pour des cloisons, ne sont que de fausses cloisons, produites par l'aplatissement l'une contre l'autre de deux extrémités polaires d'articles d'origine levuriforme, c'est-à-dire nés 
par bourgeonnement. Cette disposition se retrouve, identique, chez les levures noires. Celles-ci paraissent peu ou mal connues, aussi a-t-il paru nécessaire de résumer en quelques lignes l'état actuel de la question.

Il semble que, le premier, G. Marpmann (1886) ait décrit une levure noire, isolée du lait de vache, à laquelle il donna le nom de Saccharomyces niger (1). Cette levure a été cultivée en milieux liquides et sur gélatine où elle donnait des colonies noires, formées de cellules levuriformes et de filaments.

Avant de passer en revue les types de levures noires décrits après Marpmann, il faut citer la revue d'ensemble publiée par E. Przibram en 1925, dans laquelle il mentionne neuf types de levures noires (y compris celle de Marpmann). Ce sont les levures de Marpmann (1886), de Lindner (1897), les trois formes étudiées par Will (19131914), celles de Löwy (1922), d'Askanazy (1922), de Mahdi Hassan (1923). Mais Przibram n'avait pas eu connaissance du Monilia nigra décrit par Burri et Staub en 1909.

Avant de dresser une liste de ces divers organismes, il est tout à fait nécessaire d'établir une distinction qui a été méconnue ou insuffisamment précisée par les divers auteurs qui se sont intéressés à cette question.

Cette distinetion repose sur la nature de la filamentisation. Deux cas peuvent se présenter.

Dans le premier, il existe toujours, à un moment donné, dans les colonies complètement développées, des filaments aériens, c'est-àdire un mycelium vrai, tubuleux, cloisonné, ce qui n'empèche pas l'existence, au début, d'une phase levuriforme plus ou moins prolongée, avec élėments séparés et ébauches plus ou moins nettes de pseudomycelium. Il ne s'agit done pas d'une levure, mais d'un champignon filamenteux, hyphomycète, à thalle fuligineux, à phase levuriforme.

Dans le second cas, il n'y a jamais de filaments aériens. La filamentisation, si elle existe, est uniquement constituée par un pseudomycelium plus ou moins immergé dans le milieu solide. Il s'agit done ici d'une véritable levure, ou champignon levuriforme, lui aussi à thalle fuligineux.

La position systématique réelle de ces champignons ne peut être établie sérieusement qu'en tenant compte de cette distinction.

Voici, d'après Przibram et Will, les caractères des souches les plus anciennement connues.

(1) A cette époque, le terme générique Saccharomyces n'avait pas la signification précise qu'il possède maintenant. On s'en servait (et on s'en est encore servi longtemps) pour désigner toutes espèces de levures. 
I. Colonies fuligineuses, olivâtres ou noirâtres, présentant une phase levuriforme au début, puis recouvertes à la fin par un gazon de filaments aériens plus ou moins feutrés.

1. Saccharomyces niger Marpmann, 1886. - Isolé du lait à Weende près de Göttingen, en 1885. Colonies recouvertes à la fin d'un gazon noirâtre, velouté. Matériel étudié ensuite par E. C. Hansen (1887), puis par Guilliermond (1902), qui ont retrouvé la phase levuriforme, ainsi que le mycélium vrai, cloisonné, du type Dematium. Il ne peut donc s'agir d'un Saccharomyces, aussi Guilliermond désigne-t-il cet organisme sous le nom de Torula nigra, tandis que Hansen le rapproche des Fumago. Ces deux genres font partie des Hyphomycètes.

2. Tornla nigra de Lindner, 1887. - Souche isolée de l'air et étudiée par Lindner à Berlin, dans le laboratoire de Koch. Les colonies consistent d'abord en un coussinet noir, formé de levures bourgeonnantes, puis la surface se recouvre d'un gazon olivâtre foncé, formé de filaments. En piqûre profonde en gélatine, aspect de sapin renversé.

3. Souches de Will, 1913-1914. - Ce sont les formes I, II et III de Will. La forme I provenait de la Station zymologique de Geisenheim am Thein : colonies à surface mate, noir-verdâtre, avec mycélium aérien. La forme II provenait de l'Institut d'hygiène d'Erlangen : colonies à surface veloutée, noir-verdâtre, avec mycélium aérien. La forme III venait de l'Institut d'hygiène de Kœnigsberg: colonies à surface mate, noire, finement granulée, mycélium aérien noir, moins développé que dans les formes I et II.

Ces trois formes présentent au début une phase levuriforme, avec pseudomycélium et glomérules de blastospores, puis un mycélium vrai, fuligineux, formant gazon.

Will n'a pas donné à ces formes de dénomination précise. Il se contente de conclure qu'elles n'appartiennent ni aux Saccharomyces, ni aux Torula, ni même aux levures noires.

4. Levure noire d'Askanazy, 1922. - Souche isolée à Genève d'un contenu d'estomac. Colonies noires, plutôt mates, rarement lisses et crémeuses. Mycélium aérien noir ou brun-gris. Cellules levuriformes apiculées. Filaments conidifères.

Ces quatre types de « levures noires » sont en réalité des phases levuriformes de dématiées, c'est-à-dire de champignons à thalle fuligineux, dont le prototype est Pullularia pullulans (de Bary), Berkhout 1923 (ancien Dematium pullulans de Bary), présentant au début une forte poussée de blastospores bourgeonnantes, suivie de l'apparition d'un mycelium cloisonné et conidifère. Il convient donc de rapporter au genre Pullularia tous les champignons présentant la succession de ces deux phases. 
II. Levures véritables à colonies noires crémeuses, pas de filaments aériens, mais un pseudomycelium immergé, plus ou moins nettement verticillé.

1. Levure noire de l'air, souche de E. Löwy 1922, étudiée par Goldlust et Przibram 1925. Colonies crémeuses, noires, avec éléments levuriformes plus ou moins allongés et des ébauches de filaments.

2. Torula nigra (Monilia nigra). - Souche Mahdi Hassan, isolée à Bangalore (Inde), du sang d'un insecte des arbres à laque et auquel elle fournirait son oxygène. Colonies noires, crémeuses, à éléments levuriformes apiculés et à pseudomycélium immergé et ramifié.

Przibram propose, pour toutes les levures noires signalées dans son travail d'ensemble, le nom de Torula variabilis.

3. Torula nigra de Lindner, 1887. - Souche de Moscou, isolée à Moscou par Zeidler, sans indication précise d'origine et existant aussi dans le laboratoire de Koch, à Berlin. Colonies à surface humide, plissée et fraisée, formées d'éléments levuriformes. Pas de filamentisation en milieu liquide. Sur gélatine, à la fin, apparition d'un léger revêtement laineux.

4. Monilia nigra de Burri et Staub, 1909, et de Maurizio et Staub, 1928. -. Ces deux mémoires, publiés à 19 ans de distance, ont trait à un même champignon levuriforme, produisant des taches noires sur le fromage d'Emmenthal. Ils constituent la première étude réellement approfondie sur la question des levures noires.

Sur le fromage d'Emmenthal, ainsi que sur divers milieux de culture solides, les colonies noires, crémeuses, sont principalement constituées par des éléments levuriformes bourgeonnants ; on trouve aussi des cellules plus allongées formant, soit des ébauches de filaments, soit un pseudomycélium bien développé. Il y a aussi des arthrospores ( oïdies»), çui naissent par désarticulation des filaments, et des chlamydospores arrondies, à paroi épaisse, le plus souvent en chaînettes et séparées par des épaississements ou disjoncteurs.

Ce champignon est protéolytique, il liquéfie la gélatine et la caséine. Il est halophile et supporte un degré élevé de salure. Le chlorure de sodium paraît même, à certaines concentrations, agir comme stimulant.

La production du pigment fuligineux n'a lieu qu'en présence de l'oxygène et jamais en anaérobiose. Elle exige aussi une assez forte concentration en glycose $(25$ à 30 p. 100) et une faible concentration en éléments azotés.

5. Levures noires de Browne, 1918. - C. A. Browne, au cours d'une étude sur Jes microorganismes destructeurs du sucre de canne brut dans les entrepôts, a fait connaitre deux types de levures noires, isolées à Cuba ou dans les Indes occidentales anglaises.

a. Monilia nigra Browne, 1918. - Colonies noires, d'abord étoilées, formées de filaments constitués par des cellules bourgeonnantes, le tout 
donnant à la colonie l'aspect d'une «étoile de mer ». Plus tard il y a pullulation de cellules bourgeonnantes, recouvrant le centre de la colonie d'une masse blanche. Plus tard, enfin, les extrémités des filaments du premier stade se projettent en dehors du conglomérat de blastospores et se transforment en chapelets de chlamydospores fuligineuses, prenant une forme arborescente ou en arbuscules et une coloration noire de jais.

Si le développement des colonies s'arrête avant la formation des chlamydospores, ces colonies restent blanches, surtout lorsqu'elles sont confluentes, et alors elles recouvrent la gélose d'une couche blanche épaisse.

Les chlamydospores ont une paroi épaisse et forment des chainettes simples ou ramifiées, ayant tendance à se désarticuler. En milieu liquide, il y a formation d'un anneau filamenteux de $2 \mathrm{~mm}$.

b. Monilia fusca Browne, 1918. - Dans les sucres bruts de Cuba. Colonies plus filamenteuses que celles de $M$. nigra, ayant moins de tendance à s'étaler en couche crémeuse. Chlamydospores olivâtres, au lieu du noir de jais de $M$. nigra. En milieu liquide, anneau élevé de $3 \mathrm{~cm}$. et odeur fruitée prononcée.

Chez ces deux espèces, les filaments du pseudomycélium se couvrent de verticilles, plus ou moins confluents, de blastospores bourgeonnantes et se terminent par des chaînettes de chlamydospores fuligineuses. Ces deux organismes attaquent fortement le saccharose, qui est interverti, sauf en solutions très concentrées. $M$. fusca est moins sensible que M. nigra à la concentration des jus, aussi est-il un des plus puissants destructeurs du sucre de canne dans les entrepôts.

6. Monilia nigra. Cas de L. M. Smith et G. M. Lewis, 1936. - Ces auteurs ont rencontré, dans une lésion humaine, un champignon levuriforme noir qu'ils rapprochent du Monilia nigra de Burri et Staub. Ce serait le premier cas de mycose humaine produit par une levure noire.

Il s'agit d'une infection mycosique profonde des deux régions malléolaires, chez un homme de 67 ans, originaire de l'Ohio et habitant depuis 4 mois le New Mexico. Quand il fut examiné pour la première fois, les lésions, d'abord. nodulaires, prenaient ensuite l'aspect d'ulcérations de couleur rouge pourpre, surélevées et infiltrées, d'aspect verruqueux ou papillomateux, avec de nombreux pertuis d'où on pouvait exprimer du pus. Ces lésions rappelaient celles qui ont été décrites dans des cas de blastomycoses, chromoblastomycoses ou de tuberculose verruqueuse de la peau. Après plusieurs récidives, ces lésions finirent par céder à la röntgenthérapie et à un traitement ioduré par voie buccale.

Des cellules de levures et des ébauches de pseudomycelium ont été constatées à l'état frais dans le pus, des cultures de levures noi- 
res ont été obtenues à plusieurs reprises de différentes lésions. Il y avait pourtant, au début, une infection secondaire staphylococcique qui masquait la mycose profonde. Après amélioration de cette surinfection superficielle, on put obtenir des cultures pures de la levure noire.

Au cours de l'étude anatomo-pathologique, les auteurs ne paraissent pas avoir rencontré de Fumago, ou formes fumagoïdes, dans les microabcès des lésions fistuleuses. Ils rapportent leur levure noire au Monilia nigra et constatent que ce champignon est entièrement différent du Phialophora verrucosa de Thaxter, malgré la présence, dans les deux cas, d'un pigment fuligineux.

Disons tout de suite que l'absence de Fumago ne permet pas de ranger le cas de Smith et Lewis parmi les chromomycoses. Si la levure noire est bien l'agent causal de ces lésions, celles-ci appartiennent à un type spécial, non encore décrit auparavant.

En effet, bien que les lésions aient été fistulisées, profondes et purulentes, il n'a pas été constaté de grains. On ne peut donc.proposer le diagnostic de mycétome.

Mycétomes à Torula. - Indiquons une fois de plus la tétrade des signes caractéristiques des mycétomes (1) : tuméfaction, fistulisation, pus dans les fistules et grains dans le pus, la présence de ces derniers étant le symptòme le plus essentiel. L'attaque du système osseux est facultative, certains mycétomes, bien connus et parfaitement étudiés, ne la produisent jamais, par exemple le mycétome à grains blancs de Vincent à Nocardia maduræ, le mycétome à grains rouges à Nocardia pelletieri, etc.

Il s'est révélé une tendance regrettable à confondre, dans certains cas, mycétomes et chromomycoses. Nous venons d'énumérer les caractéristiques des mycétomes Celles des chromomycoses sont tout aussi nettes : infections purement dermiques et superficielles, sans tendance à la généralisation ; lésions verruqueuses ou couvertes de fongosités, non purulentes, mais suintantes et saignantes, débutant souvent par une hyperkératose ; dans l'épaisseur des fongosités, micro-abcès plus ou moins nombreux avec cellules géantes ; pas de grains, mais des éléments parasitaires du type Fumago, libres ou inclus dans les cellules géantes. Ces Fumago (cellules sclérotiales des mycologues nord-américains) sont la signature, la caractéristique essentielle des chromomycoses, comme le grain l'est pour les mycétomes.

(1) M. Langeron. - Les Mycétomes. Nouvelle pratique dermatologique, II, 409-456, 2 pl. Paris, Masson et Cie, 1936. 
Le cas de Smith et Lewis se rapproche beaucoup des mycétomes, mais ne peut être catalogué comme tel avec certitude. En tout cas, ce n'est pas une chromomycose, puisqu'il n'a pas été trouvé de Fumago.

Un autre cas critique est le mycétome de la main, étudié par Lewis, Hopper, Sachs, Cormia et Potelunas (1948). Ici il s'agit bien d'un mycétome, les auteurs l'ont pressenti, mais, influencés par l'opinion d'Emmons, ils ont penché du côté chromomycose.

C'est pourtant bien un mycétome, avec tuméfaction, fistulisation, suppuration discrète et petits grains noirs $(1 \mathrm{~mm}$.) anguleux et paraissant creux ; pas d'attaque osseuse. Il n'y a pas de lésions verruqueuses avec micro-abcès à Fumago.

En outre, les auteurs ont étudié avec un soin particulier le champignon qu'ils ont isolé. Ils affirment catégoriquement, et leurs microphotographies en font foi, qu'ils n'ont pas trouvé de phialides ventrues à collerette, du type Phialophora. Contrairement à l'opinion d'Emmons, ils n'ont jamais vu les conidies sortir par une ouverture de l'extrémité du conidiophore. Ils ont vu et photographié ces conidies, attachées en des points multiples des extrémités rétrécies des conidiophores; ils ont aussi constaté la présence simultanée de conidies d'âges différents. Cet appareil conidien est du type radulaspores, c'est, par conséquent, un Trichosporium.

A vrai dire, ce mycétome de la main correspond presque exactement au mycétome du pied que l'un de nous a décrit en 1928 et dont

\section{Explication de ta Planche XXXI}

FIG. 1 à 3. - Colonies de 6 semaines, du type $\mathrm{S}$, crémeuses, lisses et brillantes à divers $p \mathrm{H}$, sur gélose glycosée. $1: p \mathrm{H} 8.2: p \mathrm{H}$ 6. $3: p \mathrm{H} 3$. Les colonies 2 et surtout 3 sont entourées d'une filamentisation profonde brun clair, la masse de la colonie étant d'un brun presque noir.

FIG. 4 et 5. - Colonies de 2 mois du type R, fortement rugueuses, de face et de profil, sur gélose glycosée.

Frg. 6 et 7. - Colonies de 3 mois, provenant d'une biopsie, sur gélose glycosée, de face et de profil. Type R.

Fig. 8 et 9 . - Colonies de 15 jours, développées sur gélose glycosée à partir d'une lésion verruqueuse. La colonie $\mathrm{n}^{\circ} 9$, lisse et brillante, est entourée d'une abondante filamentisation profonde.

Fig. 10 et 12 . Colonies crémeuses, lisses et brillantes (type S), de 6 semaines, sur milieu de Lœwenstein.

Fig. 11. - Colonies du type S, lisses et brillantes, de 6 semaines, sur gélose glycosée, 
ANNALES DE PARASITOLOGIE

T. XXIV, $\mathrm{N}^{\circ 5} 5-6,1949$
Planche XXXI

(Mémoire Berger et Langeron)

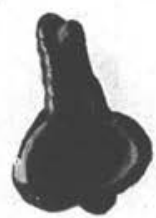

1

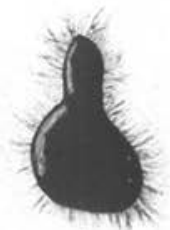

2
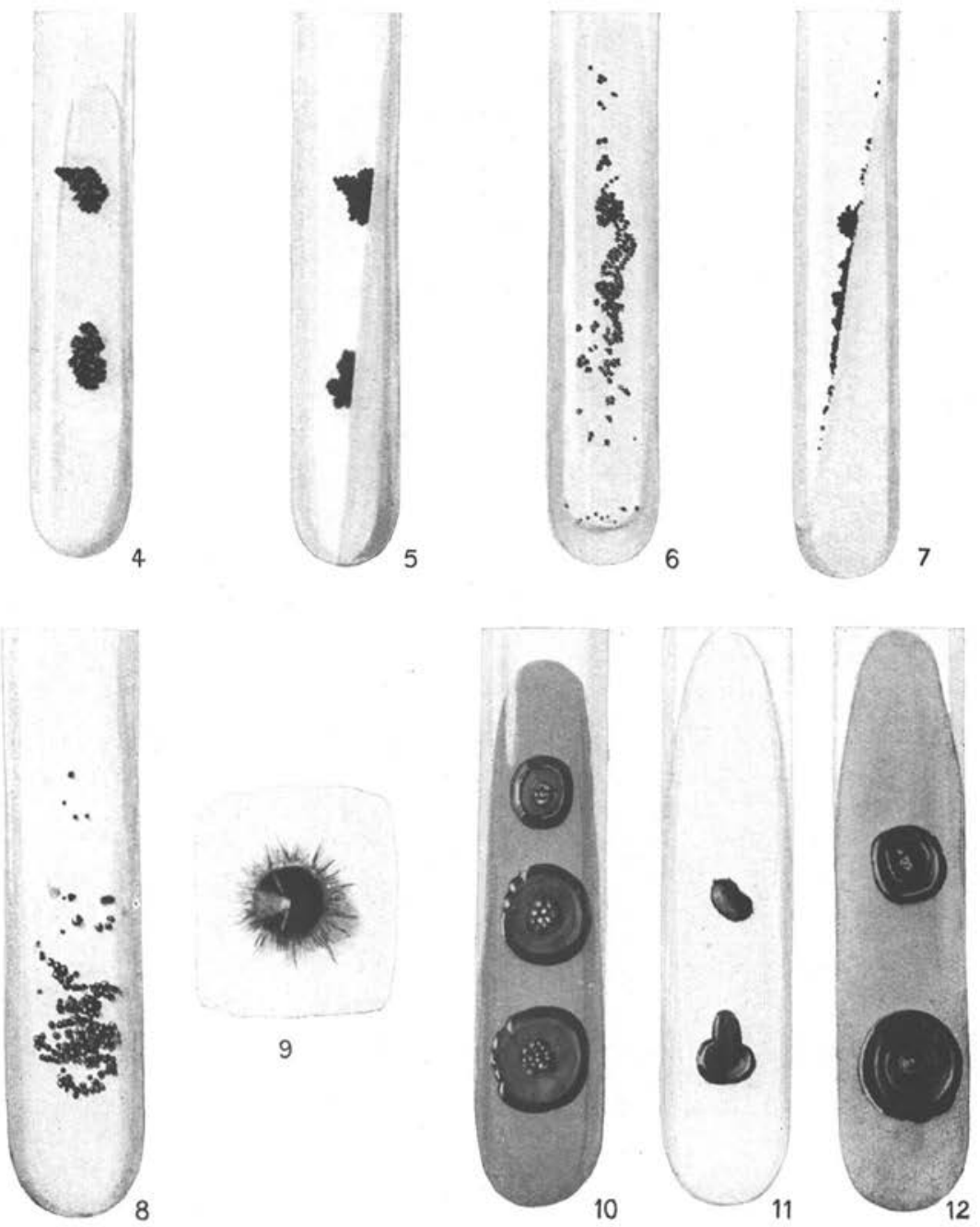

Masson et $\mathrm{C}^{10}$, Editeurs 

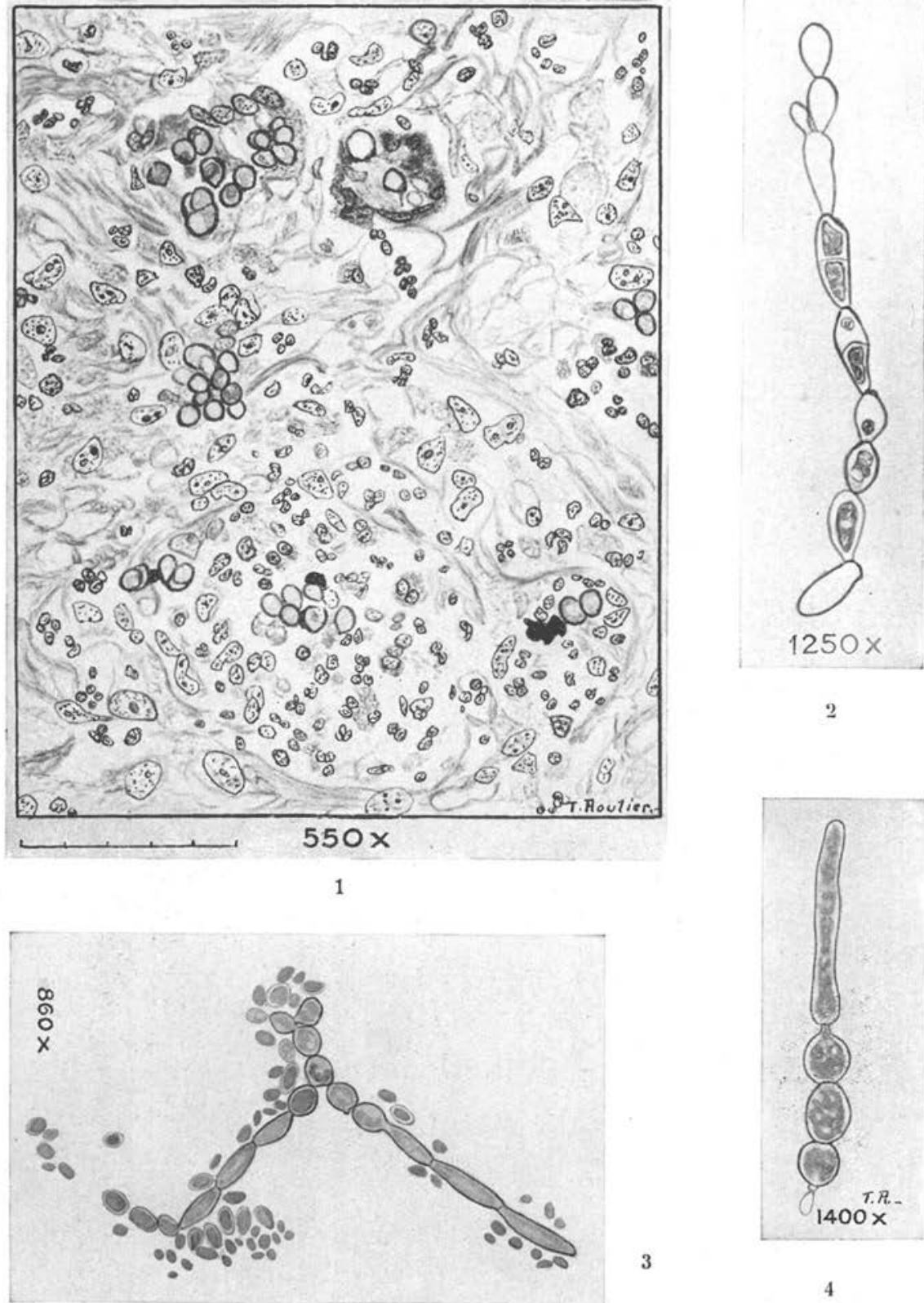

Fıg. 1. - Coupe d'une biopsie. Nombreux éléments fumagoïdes libres ou inclus dans des cellules géantes. $\times 550$.

FIG. 2, 3 et 4 . - Eléments levuriformes et pseudomycéliens développés en eau de pomme de terre. Bleu coton au lactophénol. 
ANNALES DE PARASITOLOGIE

T. XXIV, Nos 5-6, 1949
Planche XXXIII

(Mémoire Berger et Langeron)

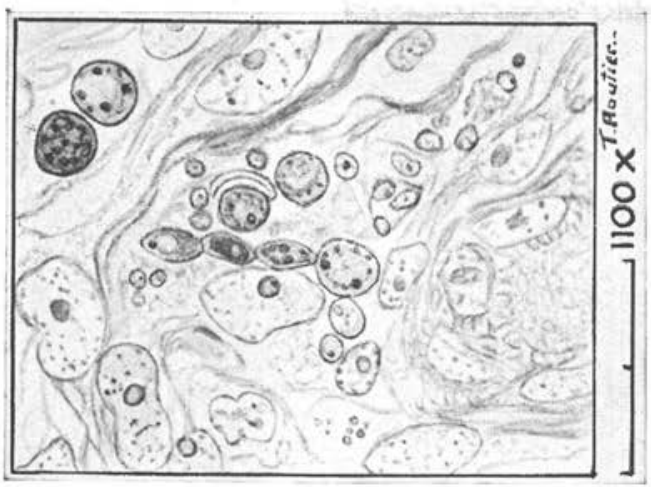

Fig. 1. - Coupe dans une biopsie. Chaînette d'éléments levuriformes dans le tissu.

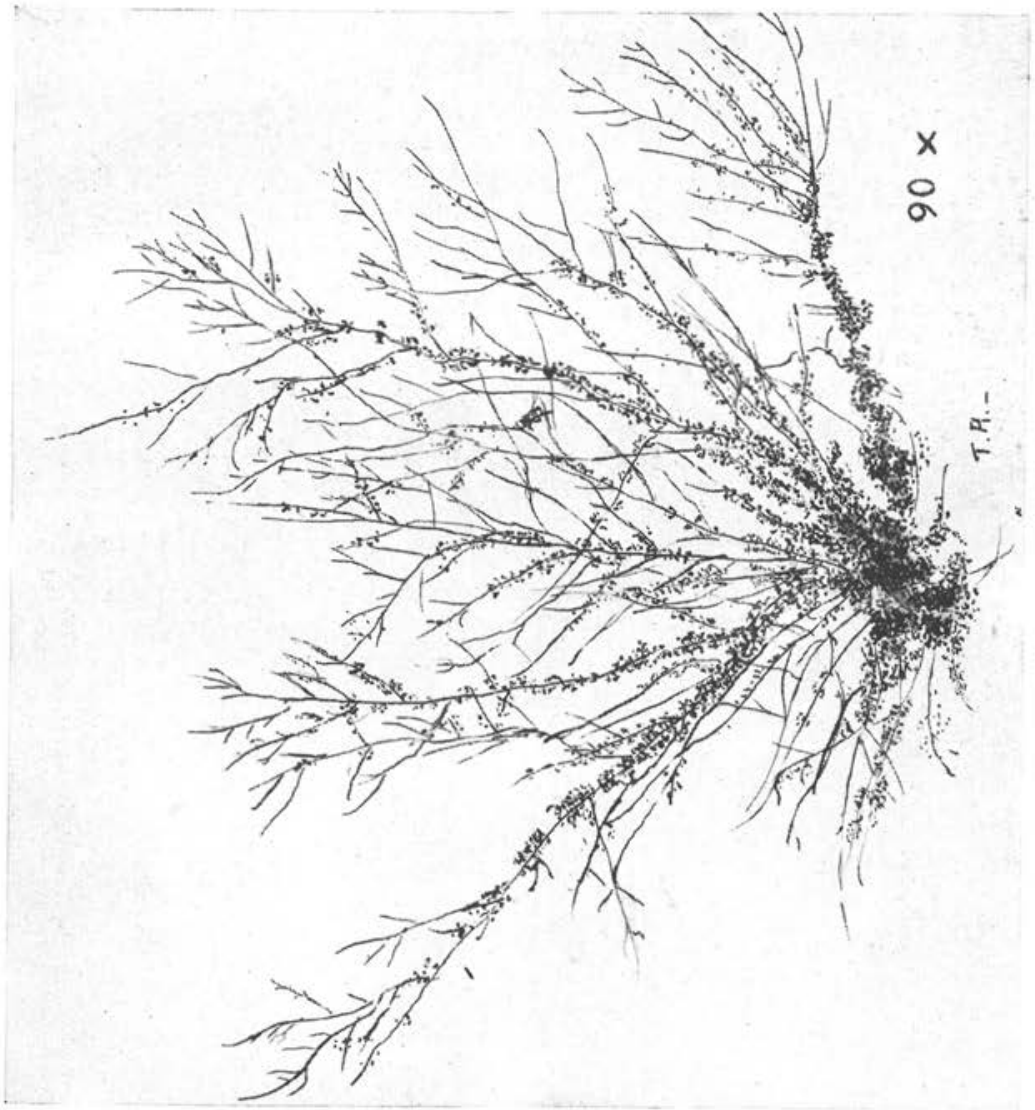

Fic. 2. - Culture sur lame gélosée, de 14 jours. Filaments ramifiés (pseudomycelium) et blastospores. $\times 90$. 
ANNALES DE PARASITOLOGIE

T. XXIV $, \mathrm{N}^{\text {os }} 5-6,1949$
Planche XXXIV

(Mémoire Berger et LANgeron)

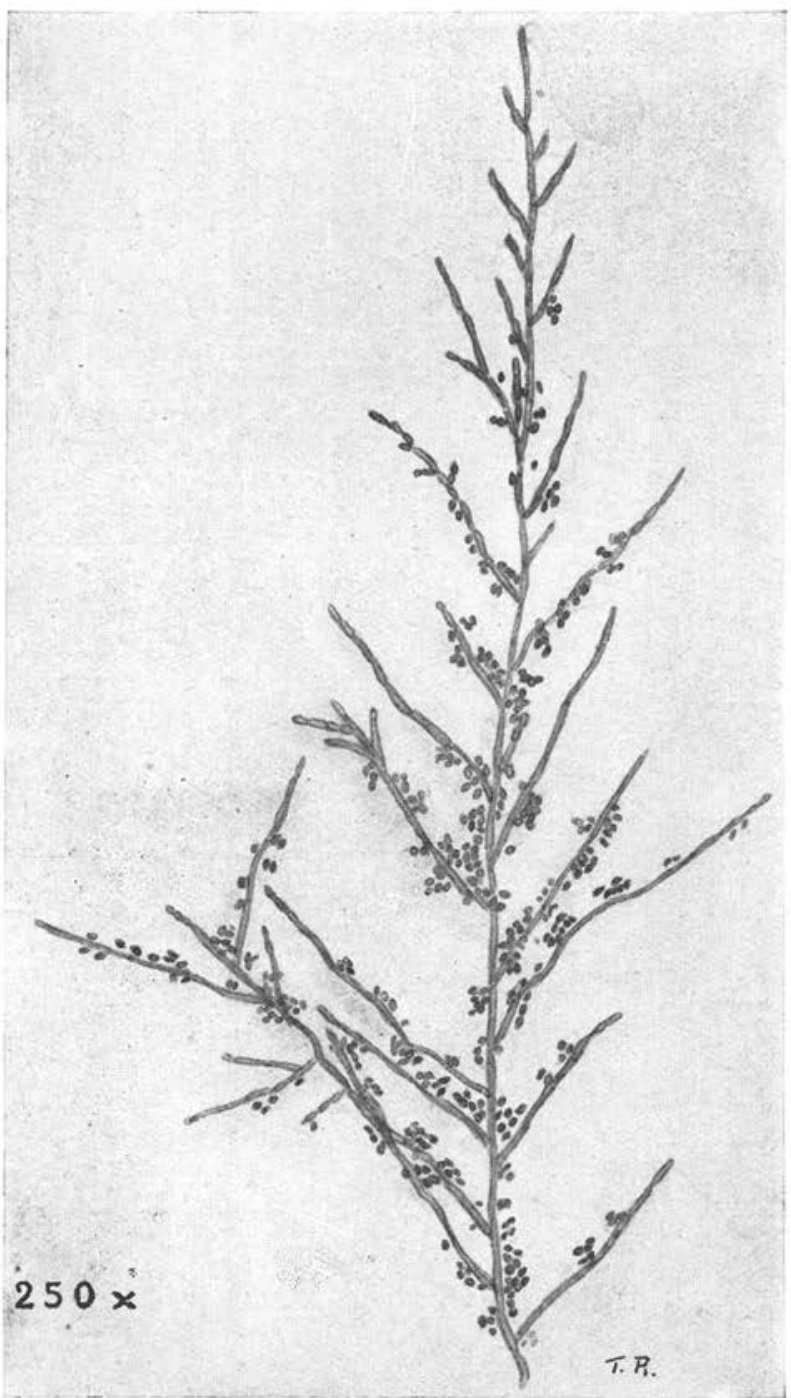

Culture sur lame gélosée de 18 jours. Pseudomyceli ım ramifié et blastospores. $\times 250$. 

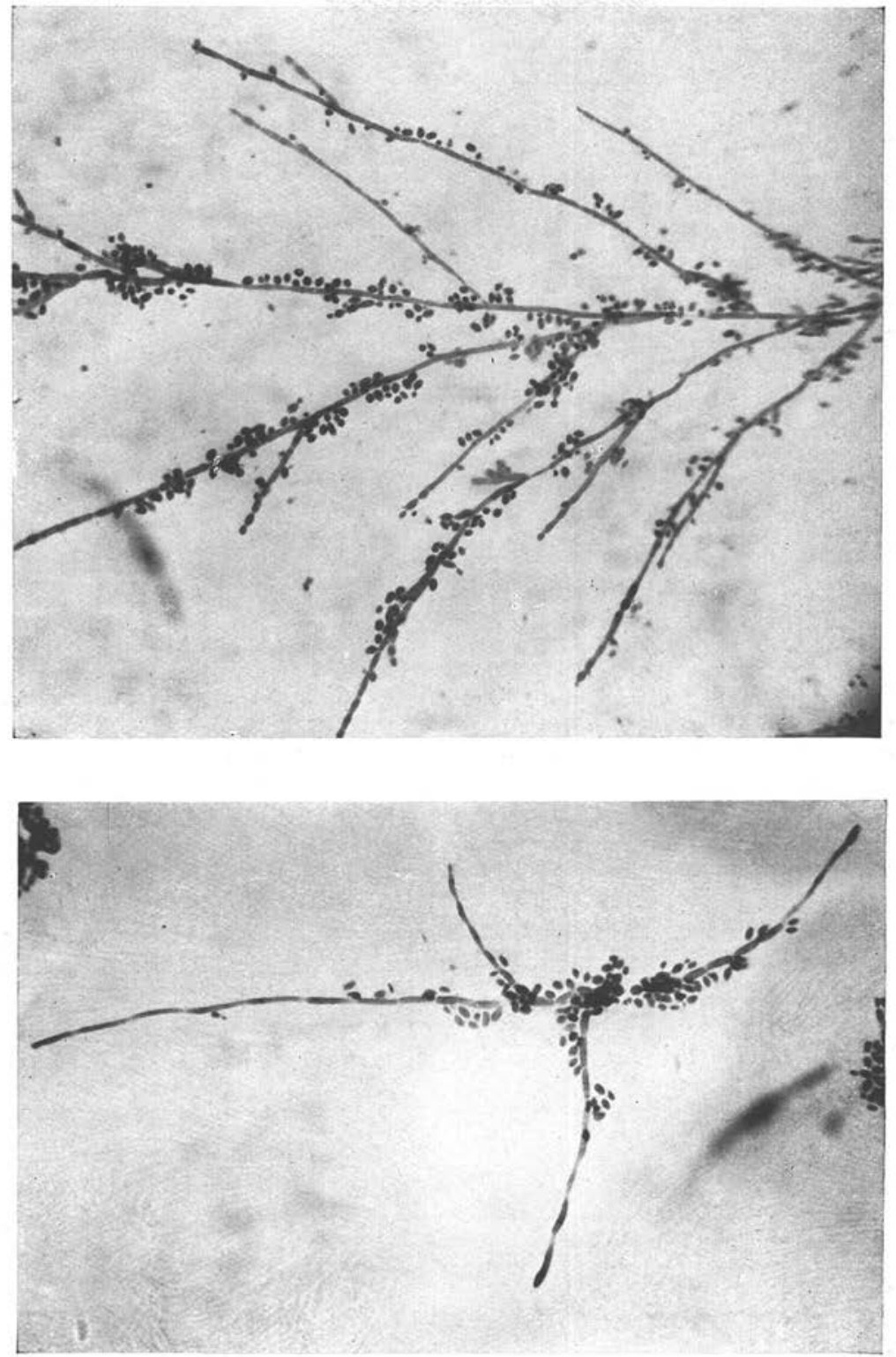

Fıg. 1. - Culture sur lame gélosée de 40 jours. Pseudomycelium ramifié et blastospores. Microphotographie $\times 370$.

Fı́. 2. - Culture sur lame gélosée de 8 jours. Blastospores et début de la filamentisation (pseudomycelium). Microphotographic $\times 420$. 


\section{LÉgende DE LA PlaNChe XXXVI}

Colonies d'environ 2 mois sur gélose glycosée. $p \mathrm{H}$ décroissant. La filamentisation est maxima entre $p \mathrm{H} 6$ et $p \mathrm{H} 4$. Elle est presque nulle à $p \mathrm{H} 6$ et $p \mathrm{H} 3$. Quand elle a lieu elle est toujours immergée dans le milieu. 
ANNALES DE PARASITOLOGIE

T. XXIV, $N^{\circ 5} 5-6,1949$
Planche XXXVI

(Mémoire Berger et Langeron)

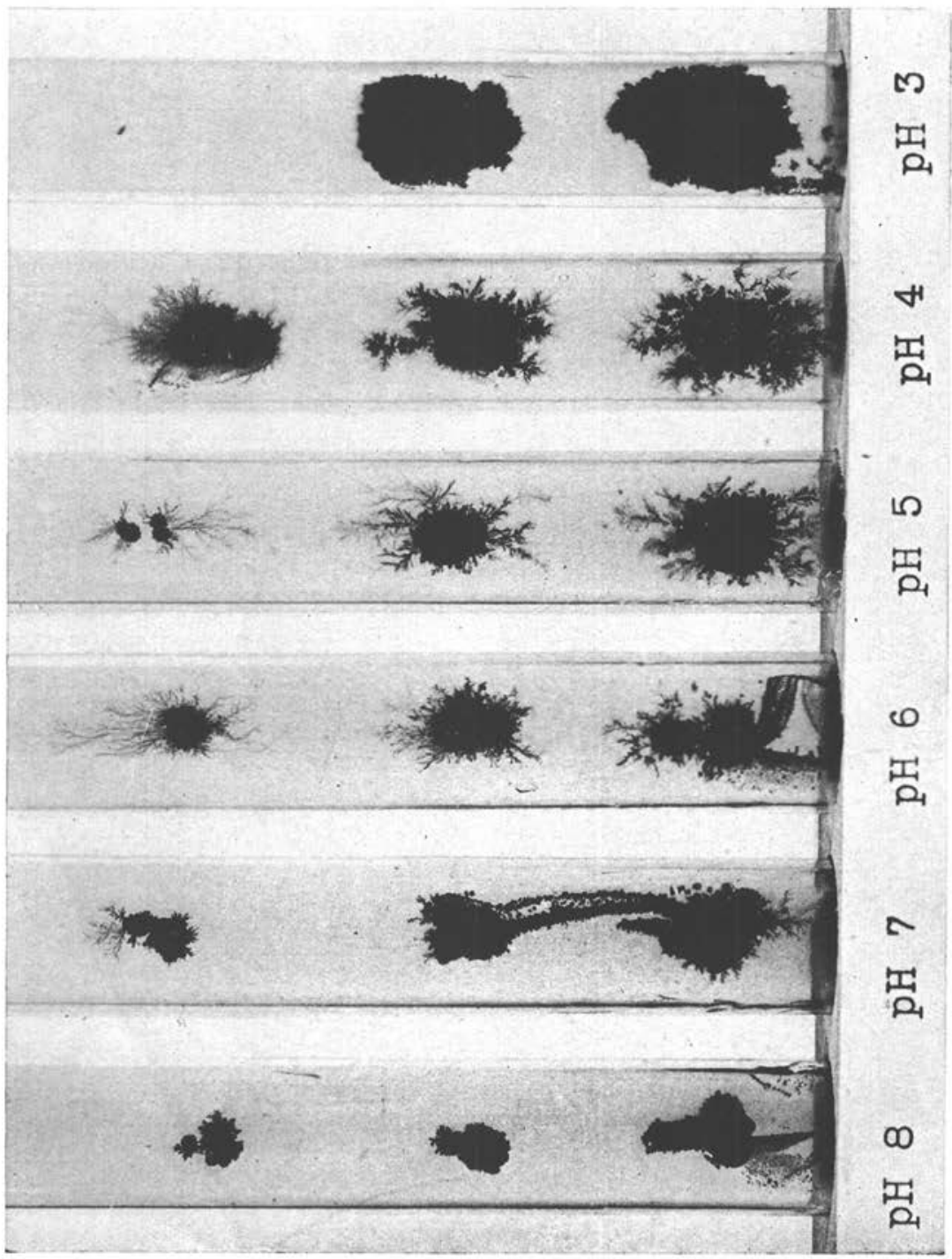




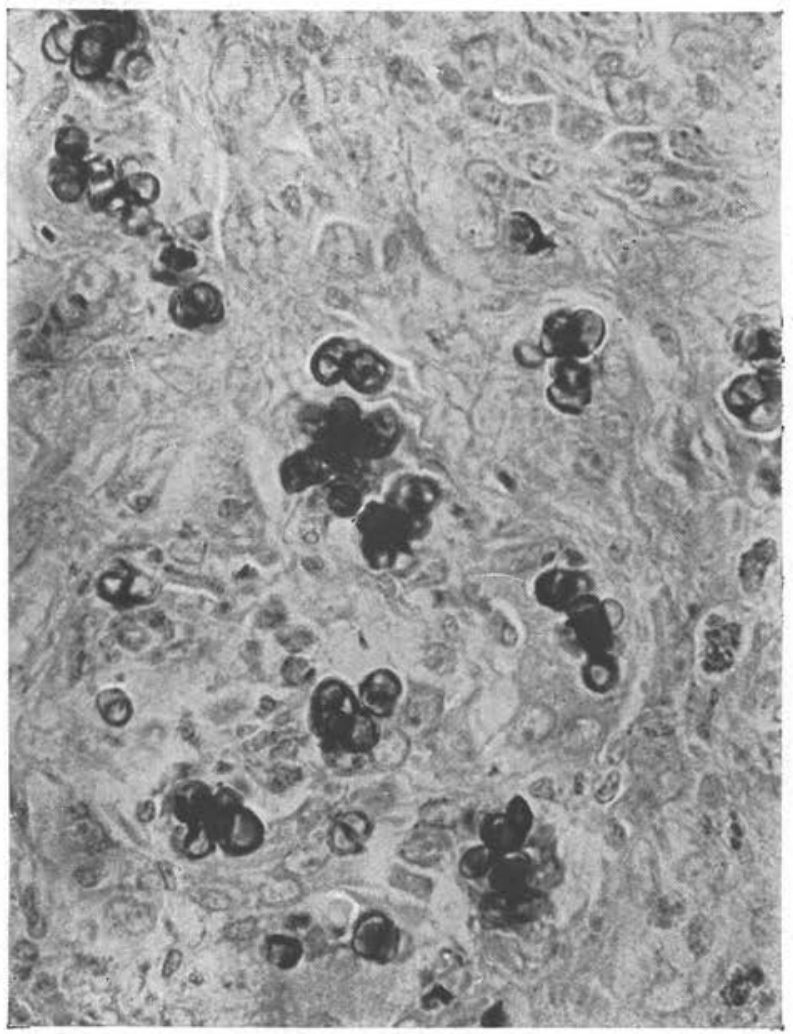

Fig. - Formes Fumago dans une des dernières biopsies (microphotographie) 
il a isolé un champignon fuligineux auquel îl a donné le nom de Torula jeanselmei. Ce champignon présente une phase levuriforme avec production intense de formes levures bourgeonnantes, comme dans le type Pullularia (1), puis apparait un mycelium vrai, cloisonné, dont les filaments portent, à l'extrémité de rameaux plus ou moins renflés, des bouquets de conidies. Dans ce cas encore, il n'y a pas, comme l'a pensé Emmons (1945), d'ouverture à l'extrémité des rameaux conidiophores, et ceux-ci ne sont pas des phialides. Les conidies sont d'origine purement externe et tombent sans laisser de trace appréciable.

Il existe un troisième cas de mycétome à Torula, c'est celui de Symmers et Sporer (1944) qui a fait l'objet de l'étude critique d'Emmons (1945). C'est aussi un mycétome de la main et il a été reproduit expérimentalement chez le lapin par Symmers (1945). Le champignon fuligineux isolé de ce cas paraît bien présenter la morphologie du Torula jeanselmei. En tout cas ce dernier ne peut absolument pas passer dans le genre Phialophora puisqu'il ne forme pas de phialides, et il y a de très grandes chances pour que les deux mycétomes de la main décrits par Symmers et Sporer et par Lewis, Hopper, Sachs, Cormia et Potelunas soient des mycétomes à Torula jeanselmei. $\mathrm{Ni}$ cliniquement, ni anatomo-pathologiquement, ni mycologiquement, on ne peut les rattacher aux chromomycoses.

Un quatrième cas a été décrit en 1948 par Pellissier, Laurent, Catanei et Montpellier. C'est un mycétome à grains noirs de la jambe, avec propagation à la face, observé à Brazzaville. Bien que les lésions aient un aspect verruqueux, elles constituent bien un mycétome et le champignon isolé ne produit pas de phialides.

Aucun de ces auteurs n'a recherché si les grains noirs de ces mycétomes avaient le caractère vermiculaire des grains de Torula jeanselmei.

Il était nécessaire de bien délimiter ces affections à cause de la confusion qui pourrait s'établir entre chromomyeoses et mycétomes et entre leurs agents.

7. Levure noire de Treillard. - $\mathrm{H}$. Treillard (1927) a décrit une levure noire provenant d'un ensemencement sur gélatine d'eau de mer prise au large de Banyuls. Les colonies étaient noires, épaisses, plissées, mates. Elles étaient surtout formées d'éléments levuriformes, le pseudomycelium étant à peu près inexistant. En milieu liquide : dépôt noir ; voile très tardif, grisâtre ; anneau épais, noir

(1) Aussi Torula jeanselmei pourrait-il être transporté dans le genre Pullularia quand on connaîtra mieux les espèces qui peuvent y être rapportées. 
foncé, humide, tardif ; traînées le long du tube. Les éléments levuriformes étaient petits $(3-5 \mu)$, à paroi très épaisse et pigmentée : le pigment est un pigment de membrane, ni intra, ni extracellulaire. Il y avait aussi des cellules géantes, à paroi mince, bourrées de lipides et de grandes cellules à coque épaisse, irrégulière, très pigmentée, à cloisons diamétrales. Les ébauches de filaments avaient des parois minces ou épaisses, peu pigmentées, grises ou brun foncé, les éléments étant chargés de lipides. On observait un bourgeonnement multiple, donnant des familles de cellules. Gélatine non liquéfiée. Probablement azymatique. Pas d'ascospores.

8. Levure noire de Schaw. - L'histoire de cette levure noire est assez bizarre. Isolée par F. W. Schaw (de l'Université de Richmond, Virginie, U.S.A.) des selles d'un malade atteint de diarrhée chronique, elle fut envoyée, en vue de détermination, au Prof. Manuel José Pereira-Filho de la Faculté de médecine de Porto-Alegre (Rio Grande do Sul, Brésil). L'étude en fut faite et publiée et, bien que les colonies fussent noires, lisses et brillantes, sur tous milieux solides, elle reçut le nom de Mycotorula schawi, sans qu'apparemment il ait été tenu compte de la pigmentation noire. Bien entendu, cette levure est azymatique. Elle donne un voile, ainsi qu'un anneau membraneux, adhérant à la paroi du tube, dans l'eau peptonée glycosée. En milieu de Lodder à l'alcool éthylique, la végétation est luxuriante, avec voile épais et anneau adhérent; le liquide se trouble. Filamentisation abondante en eau de pomme de terre. La morphologie est en tout semblable à celle des levures blanches anascosporées, ce qui explique la bizarrerie de la détermination. L'étude mériterait d'en être reprise.

\section{La chromomycose canadienne de Louis Berger}

La revue critique que nous venons de donner des "levures noires » a pour but de situer, parmi les levures noires, le nouveau champignon découvert au Canada. En effet, chez ce champignon, en dehors des Fumago, il n'a jamais été observé de mycelium vrai, mais seulement un pseudomycelium et des éléments levuriformes.

En outre, la levure noire canadienne se distingue immédiatement de toutes les autres par le fait qu'elle donne en abondance, dans les cultures, des formes Fumago identiques à celles qui existent en grand nombre dans les lésions. Ce sont ces Fumago qui donnent naissance, dans les cultures, à la phase levuriforme. Au bout d'un temps variable, cette forme s'atténue et finit par disparaitre. Les colonies perdent alors leur aspect humide et brillant, la surface. 
devient irrégulière, granuleuse et comme formée d'une poussière noire grossière, cette poussière étant constituée par les Fumago. Il se passe quelque chose d'analogue à la transformation des colonies $S$ en type $R$, au cours de ce qu'on a nommé si improprement «dissociation ».

Les Fumago ont été décrits plus haut:

Eléments cellulaires arrondis, volumineux, à paroi mince lorsqu'ils sont jeunes et se divisent activement, plus tard à paroi très épaisse, incrustée de pigment fuligineux. A ce stade, ce sont en somme de grosses * chlamydospores fonctionnelles », susceptibles, au bout d'un certain temps et dans des conditions favorables, d' "éclore » en livrant passage, soit à un jeune Fumago à paroi mince, soit au début d'un pseudomycélium. Multiplication par cloisonnement diamétral : deux cloisons méridiennes perpendiculaires divisent la petite sphère en quatre segments qui se séparent ensuite, puis s'arrondissent, et le processus recommence, de manière à constituer un amas plus ou moins volumineux de Fumago.

Il reste à savoir quel nom doit être donné à ce champignon. Il n'est pas possible de conserver le nom de Candida nigra canadensis: non seulement c'est une dénomination trinominale, mais il ne peut y avoir de Candida «noir », puisque ce nom est universellement (ou à peu près) accepté pour désigner des levures blanches anascosporées. Candida a donc une signification et une diagnose tout à fait précises.

Monilia nigra, comme on l'a vu plus haut, a été employé, par divers auteurs, pour désigner des souches présentant le caractère commun de donner des éléments levuriformes noirs ; mais ces souches sont différentes les unes des autres, encore très mal connues et on ne saurait y rattacher le nouveau champignon canadien. En outre, Monilia désigne, avec un sens tout à fait précis, les macroconidies des Stromatinia (Sclerotinia dépourvus de sclérotes différenciés) et des Neurospora. Il a été abandonné pour la nomenclature des levures blanches et ne convient pas mieux, et pour les mêmes raisons, pour les levures noires.

Il reste Torula. L'un de nous a donné, en 1928, à propos de Torula jeanselmei, une description complète du genre Torula, entendu au sens de Persoon, qui est d'ailleurs le seul plausible. Ce terme de Torula s'applique parfaitement aux levures noires, ce qui permet d'éviter la création d'un nouveau nom générique. Il permet aussi de séparer la levure noire de la chromomycose canadienne des Phialophora, agents des autres chromomycoses.

La mise au point la plus récente qui ait été publiée sur le genre 
Torula est celle de E. W. Mason, parue dans le $\mathrm{n}^{\circ} 5$ (1941) des Mycological papers, édités par le Commonwealth Mycological Institut de Kew. Ce fascicule est actuellement le dernier d'une série comprenant les Mycological papers $\mathrm{n}^{\circ \mathrm{s}} 1$ à 5 (les autres monographies publiées dans cette collection vont actuellement jusqu'au $\mathrm{n}^{\circ} 27$ ). Nous avons vu avec plaisir que nos deux publications indépendantes (Langeron et Mason) coïncident presque exactement. En somme, Torula n'est qu'un pis-aller, qui nenferme des éléments hétérogènes. Deux caractères paraissent toutefois se détacher : les éléments sporiformes sont des thallospores (blastospores ou arthrospores) et ces thallospores sont fortement colorées par un pigment fuligineux. Mason conclut aussi que les phialidés doivent en être nettement séparés à cause du processus basifrète des formations des chainettes de phialospores, tandis que, chez les vrais Torula, le processus est basifuge. Les blastosporés incolores (type Candida) s'en séparent aussi très nettement.

Tant que ces formes, dont l'étude a été si négligée jusqu'ici, ne seront pas mieux connues, il est préférable de ne pas créer de nouveaux noms de genres, à peu près fatalement destinés à tomber en synonymie.

Torula a été repris par Mlle Berkhout (1923), par l'un de nous (1928) et enfin par E. W. Mason (1941), toujours pour des champignons à pigments fuligineux et à thallospores. Ces trois publications ont été faites indépendamment et n'ont pu influer les unes sur les autres.

En particulier, il n'y a rien à modifier à ce que l'un de nous a publié en 1928. Torula reste donc le seul nom générique possible, Monilia ayant pris un sens très précis, incompatible avec la notion actuelle des Torula.

Un terme à éviter par-dessus tout est Fonsecaea, proposé par P. Negroni en 1936. C'est le protype du nomen confusum, puisqu'il s'applique à plusieurs formes conidiennes et ne peut avoir de diagnose précise. Il tombe d'ailleurs en synonymie devant Phialophora qui date de 1915 et possède donc largement la priorité. Il n'y a aucun argument valable pour rejeter Phialophora et, en tout cas, il ne peut s'appliquer au champignon canadien qui n'a jamais présenté ni phialides, ni filaments vrais. Il en est de Fonsecaea comme des autres dénominations collectives, telles que Carrionia (proposé aussi en 1939 par L. Briceño-Iragorry pour les trois formes Phialophora, Hormodendron, Trichosporium) Blastomycoides ou Gilchristia, qui sont de même à rejeter comme superflues et confuses.

L'un de nous propose donc de nommer la levure noire canadienne 
Torula bergeri et de la dédier ainsi à la mémoire de son découvreur, le très regretté Professeur Louis Berger, de Québec.

La prédominance de la phase Fumago, dans le cycle de Torula bergeri, accentue la réalité de son origine saprophytique et vraisemblablement forestière. Comme les autres chromomycoses, la maladie canadienne est produite par un saprophyte appartenant au groupe biologique des champignons des fumagines, vivant sur les feuilles et les rameaux vivants ou morts de végétaux arborescents. C'est donc encore, très probablement, une mycose forestière.

\section{Bibliographie}

Arnaud (G.). - Etudes sur les champignons des fumagines. Ann. Ecole nation. agric. Montpellier, I-III, 1910-1912 ; IX, 1910, 239-288 ; X, 1911, 211-330; XII, 1912, 1-34. Annales mycologici, VIII, 1910, 470-476.

Aroeira Neves. - Onychomycose (?) par Acrotheca pedrosoi. Brasil-medico, XLIII, $1929, \mathrm{n}^{\circ} 3,69-70$.

Berger (L.). - Etude mycologique du premier cas canadien de chromoblastomycose. Trans, Roy. Soc. Canad. (3), XXXVIII, 1944, 161.

Berger (L.), Beaudry (M.) et Gaumond (E.). - Chromoblastomycosis due to a new species of fungus (first Canadian case). Canad. med. Assoc. J., LIII, $1945,138-143$.

Berkнout (C. M.). - De Schimmelgeschlacten Monilia, Oidium, Oospora en Torula. Thèse Fac. se. Univ. Utrecht, 1923, 73 p., 4 pl.

Bretr (M. A.). - The problem of Cladosporium herbariam. Brit. mycol. Soc. Trans. (Jubilee Meeting), XXX, 1948, 141-151.

Beysa (F. H. van), - Beschreibung der in C.B.S. vorhandenen Arten der Gattungen Phialophora Thaxter und Margarinomyces Laxa, nebst Schlüssel zu ihrer Bestimmung. A. v. Leeuwenhoel, IX, 1943, 51-76.

Browne (C. A.). - The deterioration of raw cane sugar, a problem in food conservation. J. indust, engenrg. Chem., X, 1918, 178-190.

Burri (R.) et Staub (W.). - Monilia nigra als Ursache eines Falles von Schwarzfleckigkeit bei Emmenthalerkäse. Landwsch. Jahrb. d. Schweiz, XXIII, $1909,487-522$.

Briceño-Iragorry (L.). - Sobre cromoblastomycosis. Rev. clinica Louis Razetti, I, 1936, 108-128.

Carrión (A. L.). - Chromoblastomycosis. Mycologia, XXXIV; 194:, 424-441.

Carrión (A. L.) et Silva (M.). - Chromoblastomycosis and its etiologic fungi, in Biology of pathogenic fungi, édité par W. J. Nickerson, Chronica botanica, 1947, 20-62. (Annales cryptogamici et Phytopethologici, VI).

Didpexs (H. A.). - Eine neue Pilzgattung, Hyalodendron. Zirlbl. Bakt., 2 Abt., XC, 1934, 315-319.

Emmons (C. W.). - Phialophora jeanselmei comb. n. from mycetoma of the hand. Arch. pathol., XXXIX, 1945, 364-368.

Hansen (E. C.). - Allgem. Brauer. Hopfenztg., XXVII, 1887, 1109.

LANGeron (M.). - Le Trichosporium pedrosoi (E. Brumpt 1921), agent de la dermatite verruqueuse brésilienne. Ann. de paras., VII, 1929, 145-150. 
Langeron (M.). - Précis de mycologie, Paris, Masson et Cie, 1945 ; cf. p. 548-551. Mycétome à Torula jeanselmei Langeron 1928, nouveau type de mycétome à grains noirs. Ann. de paras., VI, 1928, 385-403.

Hormodendron fontoynonti Langeron 1913, agent de l'achromie parasitaire. Bull. Soc. pathol. exot., XV, 1922, 436-443.

LaUnent (M. E.). - Recherches sur le polymorphisme de Cladosporium herbarum. Ann. Inst. Pasteur, II, 1888, 558-566 et 581-603.

Lewis, Hopper, SAcis, Cormu et Potelunas. - Mycetomalike chromoblastomyeosis affecting the hand. J. invest. derm., X, 1948, 155-168.

LINDNER (P.). - Wecherischr. Braurei, IV, 1887, 853.

Mahdihassan (S.) (1). - J. sc. assoc., Maharajahs College, Vizianagaran, I, 1923, $\mathrm{n}^{\circ} 4,125$.

- Symbiounts specific of wax and pseudo lac insects. Arch. Protistenk., LXIII, 1928, 18-22, 5 fig., pl. II.

The microorganisms of red and yellow lac insects. Arch. Protistenk., LXVIII, 1929, 613-624, pl. XVII.

Mason (E. W.). - Annotated account of fungi receined at the Imperial mycological Institute, List. II, fasc. 3, general part, 69-99, 1937. - List II, fase. 3, special part, p. 113-120, 1941. Mycological fapers, $\mathrm{n}^{\circ \times} 4$ et 5 . The Commonwealth mycological Institute, Kew, Surrey.

Marpmand (G.). - Ctrlbl. allgem. Gesundheitspfl., 1886, 422.

Maurizio (A.) et Staub (W.). - Monilia nigra Burri et Staub. Weitere Untersuchungen ueber Schwarzfleckigkeit bei Emmenthalerkäse. Ctrlbl. Balit., 2 Abt., LXXV, 1928, 375-404.

Melin (E.) et NanNfeld (J. A.). - Researches into the blueing of ground woodpulps. Svenska Skogswards Tidskrift, 1934, Häfte III, IV, 436-442.

Pellissier, Laurent, Catanei et Montpeldier. - Un cas de inycétome à Torula jeanselmei. Cahiers méd. Union française, III, 1948, 205-207.

Planchos (L.). - Influence de divers milieux chimiques sur quelques champignons du groupe des Dématiées. Ann. Sc. nat. Bot. (2), VIII, 1900, 1-248.

Pereira-Filho (M. J.). - Mycotorula schavi n. sp. Revista de med. do Rio Grande do Sul, V, 1948, $\mathrm{n}^{\circ} 26,14$ p., 5 pl.

Przibram (E.). - Ueber «schwarze Hefen » (Zymonemata nigra und eine Torula variabilis). Ergebn. Physiologie, XXIV, 1925, 95-106.

Symmers (D.). - Experimental reproduction of maduro-mycotic lesions in rabbits. Arch. pathol., XXXIX, 1945, 358-363.

Simsox (F. W.). - Chromoblastomycosis. Some observations on the types of the disease in South Africa. Mycologia, XXXVIII, 1946, 432-449.

Simson, Harington et Bannetson. - Chromoblastomycosis. J. path bact., LV, 1943, 191-198.

SMrth (L. M.) et LEwis (G. M.). - Deep mycotic infection caused by Monilia nigra. Southern med. f., XXIX, 1936, 1067-1070.

Sмiтн (L. M.). - J. amer. med, assoc., CXVI, 1941, 200-204.

Srmmers (D.) et Sporer ( ). - Maduromycosis of the hand, with special reference to heretofore undescribed foreign body granuloma formed around disintegrated chlamydospores. Arch. path., XXXVII, 1944, 309318.

(1) Ecrit au Palais du Maharaja Sir Kishun Pershad, Hyderabad, Decean, 
Terra (F.), Torres (M.), da Fonseca (O.) et de Arêa Leão. - Novo tipo de dermatite verrucosa, mycose por Acrotheca. Brasil-medico, XXXVI, 1922, $\mathrm{n}^{\circ} 49,363-368$.

Thaxter in Medear (E. M.). - A new fungus, Phialophora verrucosa pathogenic for man. Mycologia, VII, 1915, 200-203.

Тном (Ch.). - Naming Molds. J. Washington Acad. Sc., XXX, 1949, 49-64.

Tremlard (M.). - Caractères généraux et position systématique d'un champignon nouveau voisin des levures. C.R. Soc. biol., XCVII, 1927, 1699.

- Weidmax (F. D.) et Rosenthal (L. H.). - Chromoblastomycosis. A new and important blastomycosis in North America. Report of a case in Philadelphia. Arch. derm. syphil., XLIII, 1941, 62-84.

Wir. (H.). - Beiträge zur Kentnis der sogenannten schwarzen Hefen. Ctrlbl. Bakt., 2 Abt., XXXIX, 1913-1914, 1-26.

Laboratoire d'Anatomie pathologique, Faculté de médecine de l'Université Laval, Québec (Directeur : Prof. Louis Berger). Institut de Parasitologie de la Faculté de Médecine de Paris

(Direcleur: Prof. H. Galliard). Section de mycologie (Chef de service: $D^{\text {r }} M$. Langeron) 